\title{
Yielding processes in a colloidal glass of soft star-like micelles under large amplitude oscillatory shear (LAOS)
}

Frédéric Renou, Jörg Stellbrink, and George Petekidis

Citation: Journal of Rheology 54, 1219 (2010); doi: 10.1122/1.3483610

View online: https://doi.org/10.1122/1.3483610

View Table of Contents: http://sor.scitation.org/toc/jor/54/6

Published by the The Society of Rheology

\section{Articles you may be interested in}

New measures for characterizing nonlinear viscoelasticity in large amplitude oscillatory shear

Journal of Rheology 52, 1427 (2008); 10.1122/1.2970095

A geometrical interpretation of large amplitude oscillatory shear response Journal of Rheology 49, 747 (2005); 10.1122/1.1895801

Describing and prescribing the constitutive response of yield stress fluids using large amplitude oscillatory shear stress (LAOStress)

Journal of Rheology 57, 27 (2013); 10.1122/1.4754023

A sequence of physical processes determined and quantified in LAOS: Application to a yield stress fluid

Journal of Rheology 55, 435 (2011); 10.1122/1.3544591

Oscillatory yielding of a colloidal star glass

Journal of Rheology 55, 733 (2011); 10.1122/1.3579161

A sequence of physical processes determined and quantified in large-amplitude oscillatory shear (LAOS): Application to theoretical nonlinear models

Journal of Rheology 56, 1 (2012); 10.1122/1.3662962

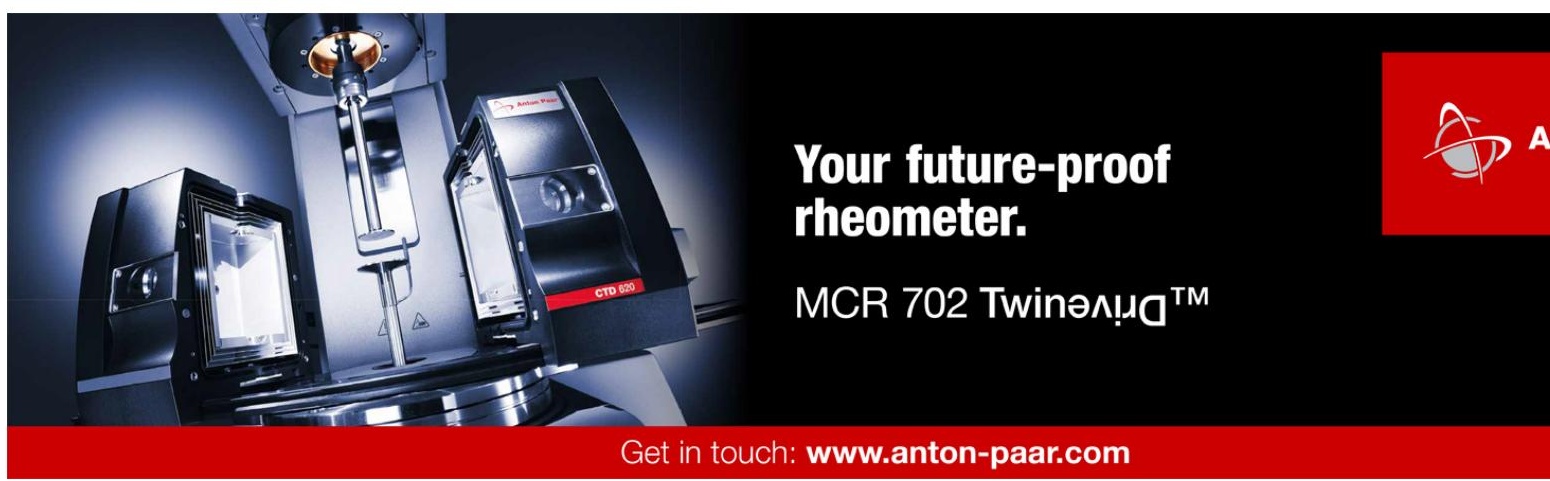




\title{
Yielding processes in a colloidal glass of soft star-like micelles under large amplitude oscillatory shear (LAOS)
}

\author{
Frédéric Renou \\ Institute of Electronic Structure and Laser, FORTH, Heraklion, Crete, Greece
}

Jörg Stellbrink

Institute für Festkörperforschung, FZ Jülich, Jülich D-52425, Germany

George Petekidis ${ }^{\text {a) }}$

Institute of Electronic Structure and Laser, FORTH, Heraklion, Crete, Greece and Department of Materials Science and Technology, University of Crete, Heraklion, Greece

(Received 12 January 2010; final revision received 4 August 2010; published 5 October 2010)

\begin{abstract}
Synopsis
The understanding of yielding and flow of a colloidal glass under large amplitude oscillatory shear (LAOS) represents a motivating challenge. Monitoring the higher harmonics in the stress signal by Fourier-transform (FT) rheology may provide useful insight on the progressive transition from linear to nonlinear viscoelastic response. However, the physical interpretation of FT-rheology data is still not obvious. Here we study the process of yielding in a colloidal glass formed by star-like block copolymer micelles with LAOS experiments and interrogate the nonlinear intracycle stress response by FT analysis and decomposition to an orthogonal set of Chebyshev polynomials [Ewoldt, R. H., et al. J. Rheol. 52(6), 1427-1458 (2008)]. Such approach provides a robust framework enabling us to map out a rich phenomenology of intracylce nonlinearities that may relate to distinct physical mechanisms. We find that the nonlinearities during yielding are represented by intracylce shear thickening/thinning and strain hardening/softening of the viscous and elastic response of the system, respectively. We suggest that the underlying mechanisms are related to cage breaking and reformation as well as stress storing and relaxation within the period of oscillatory shear which are affected by an interplay between shear and Brownian motions and thus relate to Péclet number variation with strain and frequency. (C) 2010 The Society of Rheology. [DOI: 10.1122/1.3483610]
\end{abstract}

\section{INTRODUCTION}

Colloids at high volume fractions may form metastable states where particles are kinetically trapped either due to excluded volume, entropic effects, or attractive interactions with the former usually characterized as colloidal glasses while the latter as colloi-

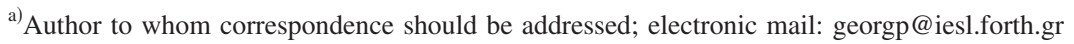


dal gels [Pusey and Van Megen (1986); Pusey and Van Megen (1987); Zaccarelli (2007)]. When both effects contribute to the dynamic arrest, the system considered an attractive glass [Pham et al. (2002)]. Due to such interactions particle diffusion over a long distance is increasingly slowed down as the volume fraction or attraction gets higher, and a two step diffusion process appears with a fast relaxation time corresponding to the diffusion on the interparticle distance or within the cage and a slow one related to diffusion over longer distances [Pusey (1991)].

The mechanical response of such glassy states is that of a viscoelastic yield stress material [Mason et al. (1996); Cloitre et al. (2003); Petekidis et al. (2003); Helgeson et al. (2007); Pham et al. (2008)] with low elastic modulus and yield stress due to the micrometric size of the constituents (thus the term soft glasses). A shear induced solid- to liquid-like transition (yielding) has been studied in a variety of such soft matter systems both under steady and oscillatory shear [Mason et al. (1996); Petekidis et al. (2002); Petekidis et al. (2003); Helgeson et al. (2007); Pham et al. (2006, 2008); Carrier and Petekidis 2009]. The goal to identify the underlying structural changes responsible for such transitions is partly achieved for model systems such as repulsive (hard sphere) and attractive glasses, where yielding is related to breaking of entropic cages [Petekidis et al. (2002, 2003, 2004)] or interparticle bonds and attractive cages [Pham et al. (2006, 2008)], respectively. In these cases, the associated yield strains are to a large extent dictated by the structural details of the system.

At high volume fraction soft colloids such as polymeric micelles [Hamley (1998); Yamazaki et al. (2002); van Ruymbeke et al. (2010)], core-shell and simple microgels [Deike et al. (2001); Le Grand and Petekidis (2008); Carrier and Petekidis (2009); Siebenbürger et al. (2009); Purnomo et al. (2008)] and star polymers [Kapnistos et al. (2000); Loppinet et al. (2001); Christopoulou et al. (2009)] exhibit liquid to solid transition. The effective volume fraction of these soft colloids may be tuned by either increasing the number density or by varying the temperature [Kapnistos et al. (2000); Renou et al. (2007); Deike et al., 2001; Carrier and Petekidis (2009)], although these two ways are not necessarily equivalent [Le Grand and Petekidis (2008); van Ruymbeke et al. (2010)]. In systems with soft repulsive interactions such as migrogels [Cloitre et al. (2003)], multiarm stars [Helgeson et al. (2007); Christopoulou et al. (2009)], core-shell particles [Le Grand and Petekidis (2008)], emulsions [Mason et al. (1996)], or foams [Rouyer et al. (2008)], yielding is also affected by particle (or outer shell) elasticity, especially for volume fractions around and above close packing [Mason et al. (1996); Seth et al. (2006); Le Grand and Petekidis (2008); Carrier and Petekidis (2009)].

Since soft particles exhibit certain similarities with regard to their dynamics and rheology [Likos (2006); Watanabe et al. (1997); Watanabe et al. (2001); Mortensen et al. (2002)], hard-sphere simple models for the latter are often employed [Senff et al. (1999); Siebenbürger et al. (2009)]. Nevertheless, such an approach fails at high volume fractions when interactions become important and affect the macroscopic properties [Le Grand and Petekidis (2008)] or for particles with long grafted chains [van Ruymbeke et al. (2010)].

On the theoretical side, there are simple phenomenological models [Derec et al. (2003); Craciun et al. (2003); Carrier and Petekidis (2009)] and mesoscopic theories [Sollich (1998); Fielding et al. (2000)] that capture main features of non-linear rheological tests. On the other hand, much more rigorous approaches such as mode coupling theory were extended to describe steady [Fuchs and Cates (2003, 2009)] and oscillatory shear non-linear tests [Miyazaki et al. (2006)] Still, however, the detailed physical mechanisms responsible for yielding in colloidal systems and the way this is affected by interparticle interactions is not fully disclosed. 
One aspect of the yielding processes is the appearance of significant non-linearities as demonstrated by a distorted stress response under oscillatory shear strain [Gadala-Maria and Acrivos (1980); Kallus et al. (2001); Derec et al. (2003); Craciun et al. (2003); Le Grand and Petekidis (2008); Carrier and Petekidis (2009)]. Thus a significant number of yielding studies on soft matter glasses has utilized large amplitude oscillatory shear (LAOS) experiments [Wilhelm et al. (1998); Nicolai and Benyahia (2005); Hyun et al. (2006); Le Grand and Petekidis (2008); Rouyer et al. (2008); Lonetti et al. (2008)]. The stress distortion observed within an oscillation cycle can be analyzed via a Fourier transform analysis (FT) calculating the higher odd harmonics contribution as a first measure for the progressive transition from linear to nonlinear behavior [Wilhelm et al. (1998); Neidhöfer et al. (2003)].

LAOS data may also be represented by the so called Lissajous plot, where the total stress is plotted versus the applied strain within a cycle [Philippoff (1966); Tee and Dealy (1975)]. Nonlinearity in the stress response results in distortion of the elliptical shape of the Lissajous plot and appearance of higher harmonics in the Fourier spectrum. Although FT rheology is widely employed, it still lacks clear physical interpretation that may relate the macroscopic rheological response during yielding with microscopic physical mechanisms. More recently, the method of stress decomposition [Cho et al. (2005)], which uses symmetry arguments to decompose the total nonlinear stress into a superposition of an elastic and a viscous contribution, was extended by Ewoldt et al. (2008) with the use of a unique set of orthogonal, first kind Chebyshev polynomials. This method provides a basis for a physically meaningful interpretation of different kinds of nonlinearities involved in yielding such as temporary strain hardening (or softening) and shear thickening (or thinning) within a cycle of a LAOS experiment.

Here, we study suspensions of soft colloidal micelles obtained by dissolving the amphiphilic diblock copolymer poly(ethylene-alt-propylene)-poly(ethylene oxide) (PEPPEO) in heavy water $\left(\mathrm{D}_{2} \mathrm{O}\right)$. Highly asymmetric PEP-PEO may form spherical star-like micelles in aqueous media [Lund et al. (2004)], which recently have been introduced as tunable analogs of regular star polymers [Laurati et al. $(2005,2007)]$. We investigate yielding and flow of such soft colloidal glass as a function of strain and frequency using both the FT analysis and the orthogonal stress decomposition. In particular, the latter leads to a physical interpretation of the intracycle nonlinearities enabling us to show that the yielding process dwells on a wide range of strain $(5 \%-50 \%)$ and involves a combination of intracycle shear-thickening/thinning and/or strain hardening/softening phenomena that depend on both the strain amplitude and frequency and may be attributed to microscopic mechanisms such cage breaking, relaxation, and reformation.

\section{EXPERIMENT}

\section{A. Materials and methods}

PEP-PEO copolymers were prepared by a two step anionic polymerization [Poppe et al. (1997)]. The number-average molar masses $\left(M_{n}\right)$ determined by size exclusion chromatography in THF/DMA at $40{ }^{\circ} \mathrm{C}$ were $1300 \mathrm{~g} / \mathrm{mol}$ for the PEP block and 20300 $\mathrm{g} / \mathrm{mol}$ for the PEO block corresponding to an overall $M_{n}=21600 \mathrm{~g} / \mathrm{mol}$ for the block copolymer. Their polydispersity indices $\left(M_{\mathrm{w}} / M_{n}\right)$ were 1.04 and 1.08 , respectively. Clear solutions of PEP-PEO were obtained in deuterated water $\left(\mathrm{D}_{2} \mathrm{O} 99.8 \%\right.$, purchased by Armar Chemicals) after stirring at room temperature for at least $3 \mathrm{~h}$. The solutions were left at measurement temperature for 1 day to equilibrate before measuring. A previous study [Willner et al. (2001); Lund et al. (2006)] has demonstrated that, in aqueous solution, PEP-PEO forms micelles where no kinetic exchange of block copolymers be- 
tween different micelles is possible due to the high interfacial tension between PEP and water $\left(46 \mathrm{mN} \mathrm{m}^{-1}\right)$. Moreover, the micelles remain stable under shear as evidenced by Rheo-SANS measurements [Stellbrink et al. (2008)]. Such micelles are called "frozen" micelles in contrast with "living" micelles where the functionality (i.e., number of block copolymers or "arms" per micelle) may vary with concentration [Renou et al. (2008)] and/or temperature [Sommer and Pederson (2004)]. Thus, for the system studied here, the absence of kinetic exchange ensures that the functionality remains constant with varying concentration and temperature.

Since $\mathrm{D}_{2} \mathrm{O}$ is a marginal solvent for PEO, the solvation of the corona changes with temperature making the micelles shrinking with increasing temperature. The inset of Fig. 1(a) shows the temperature dependence of the hydrodynamic radius $\left(R_{H}\right)$ of the micelles determined by dynamic light scattering in very dilute regime. $R_{H}$ was about $36 \mathrm{~nm}$ at $20{ }^{\circ} \mathrm{C}$, slightly bigger than in previous small angle neutron scattering measurements (SANS) in water with similar block copolymer [Lund et al. (2006)]. The $R_{H}$ decreases linearly with increasing temperature in the range of temperature investigated here [inset of Fig. 1(a)]. The functionality, $f$ (i.e., number of block copolymers or "arms" per micelle). determined by SANS was found to be around 120 and independent of temperature in agreement with [Lund et al. (2006)]. Using the values of $R_{H}$ at $20{ }^{\circ} \mathrm{C}$ and $f$, we found that the overlap concentration, $c^{*}=3 f M_{\mathrm{w}} /\left(4 \pi R_{H}^{3} N_{a}\right)$ (where $N_{a}$ is the Avogadro number) is $c^{*}=21.7 \mathrm{~g} / 1$.

\section{B. Dynamic light scattering}

Conventional dynamic light scattering (DLS) and multi-speckle dynamic light scattering (MSDLS) experiments were performed to characterize the particles and measure their dynamics in dilute and concentrated, glassy suspensions, respectively. The samples were thermostated at the desired temperature $\left( \pm 0.1^{\circ} \mathrm{C}\right)$, using a home-made temperature controlled bath. DLS and MSDLS measurements were performed with a home-made goniometer comprising of a charge-couple device (CCD) based detection for the slow dynamics and a single-mode fiber detection unit for the fast part. The laser source was a $\mathrm{Nd}$ :YAG solid state laser $\left(\lambda_{0}=532.5 \mathrm{~nm}\right)$ and maximum power of $150 \mathrm{~mW}$ with a collimated incident beam of $0.8 \mathrm{~mm}$ diameter. The collection optics images a cylindrical portion of the scattering volume $\left(0.8 \times 2 \mathrm{~mm}^{2}\right)$ onto the CCD detector; all data shown here were measured at $\theta=90^{\circ}$, corresponding to $q=0.0251 \mathrm{~nm}^{-1}\left(q R_{H} \sim 0.9\right)$. We process the CCD data using the time resolved correlation method [Cipelletti et al. (2003)], which allows us to follow both stationary and time-varying dynamics through the instantaneous degree of correlation, $c_{I}$, between pairs of images of the speckle pattern scattered by the sample at time $t_{\mathrm{w}}$ and $t_{\mathrm{w}}+\tau, c_{I}\left(t_{\mathrm{w}}, \tau\right)=\left(\left\langle I_{p}\left(t_{\mathrm{w}}\right) I_{p}\left(t_{\mathrm{w}}+\tau\right)\right\rangle_{p} /\left\langle I_{p}\left(t_{\mathrm{w}}\right)\right\rangle_{p}\left\langle I_{p}\left(t_{\mathrm{w}}+\tau\right)\right\rangle_{p}\right)-1$. Here $I_{p}$ is the intensity at pixel $p$ and the average is over all the CCD pixels. Data are corrected for the uneven illumination as in [Duri et al. (2005)]. For non-stationary dynamics (e.g., during sample aging), the two-time intensity correlation function, $g_{2}\left(t_{\mathrm{w}}, t_{\mathrm{w}}+\tau\right)-1$, is obtained by averaging $c_{I}\left(t_{\mathrm{w}}, \tau\right)$ over $t_{\mathrm{w}}$, choosing a time window short enough for the dynamics not to evolve significantly. For stationary dynamics (e.g., when equilibrium is reached), time invariance is fulfilled and $g_{2}\left(t_{\mathrm{w}}, t_{\mathrm{w}}+\tau\right)-1$ reduces to the usual intensity correlation function $g_{2}(\tau)-1$. The intensity correlation function measured by the CCD was multiplied by some constant number to rescale with the one obtained by point-like [PMT detector] which measures the fast dynamics $(\tau<10 \mathrm{~s})$, averaged over a large number of speckles through a slow rotation of the sample cell. 

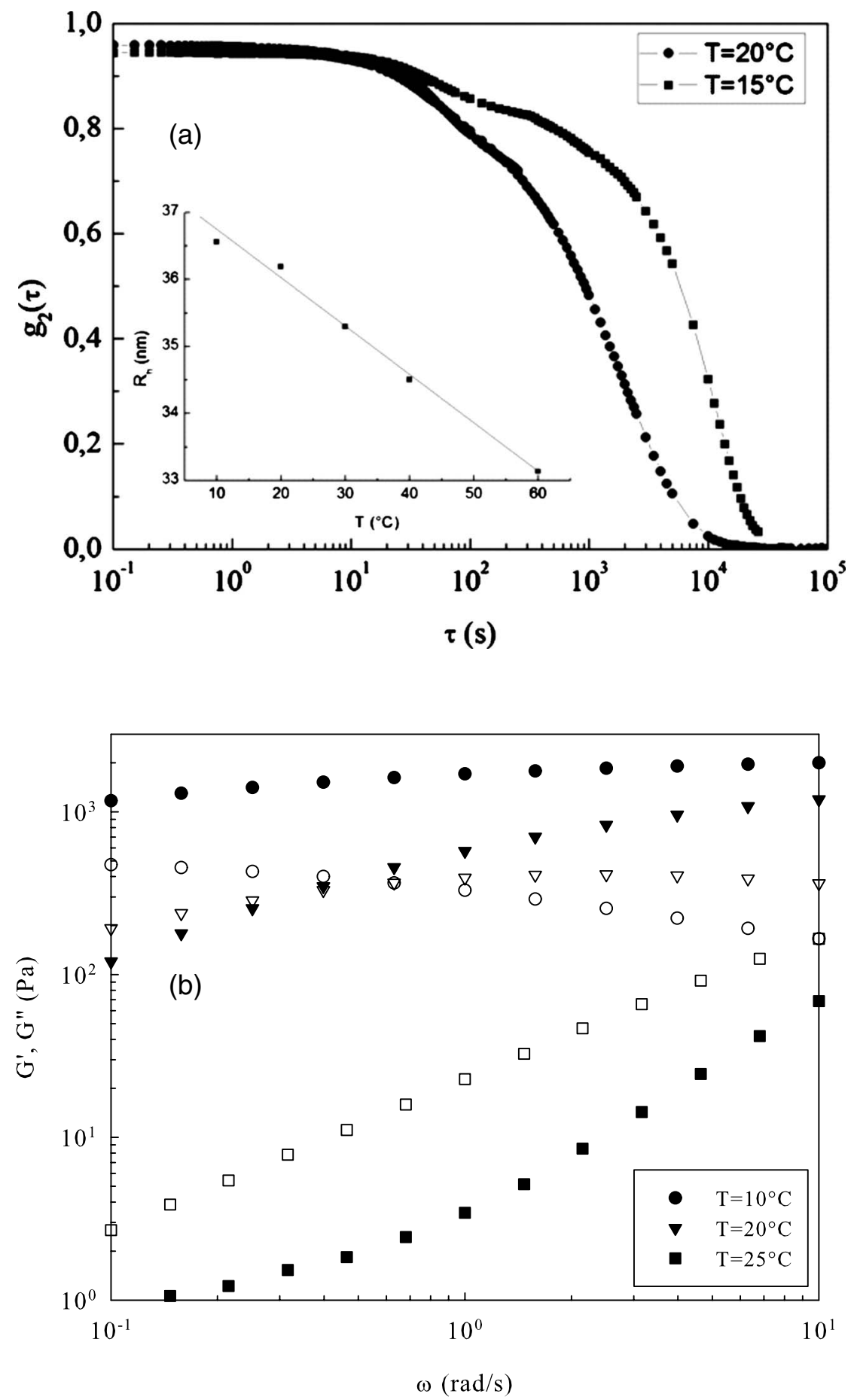

FIG. 1. (a) Intensity correlation function of a solution of PEP-PEO micelles with concentration $54.25 \mathrm{~g} / 1$ measured by MS-DLS at $\theta=90^{\circ}$ at 15 and $20^{\circ} \mathrm{C}\left(2.5 c^{*}\right)$. The inset represents the temperature dependence of the hydrodynamic radius $\left(R_{H}\right)$ from dilute solution $\left(c=4.4 \cdot 10^{-3} \mathrm{~g} / 1\right)$. (b) Dynamic frequency sweeps at $\gamma_{0}$ $=0.5 \%$ for a solution of PEP-PEO with $54.25 \mathrm{~g} / 1$ for different temperatures as indicated. $G^{\prime}$ are indicated with closed and $G^{\prime \prime}$ with open symbols. 


\section{RHEOLOGY}

The oscillatory measurements were performed in a strain-controlled rheometer ARES (Rheometric Scientific) with a force rebalance transducer 100FRTN1 in cone plate geometry ( $25 \mathrm{~mm}$ diameter and cone angle of $0.048 \mathrm{rad}$ ). Dynamic strain sweep experiments were performed at different frequencies to determine the elastic and viscous moduli $G^{\prime}$ and $G^{\prime \prime}$ as a function of strain amplitudes varying from $0.1 \%$ to $100 \%$. Dynamic time sweeps were performed at specific strains and frequencies in the range of $0.1 \%$ to $500 \%$ and $0.1-20 \mathrm{rad} / \mathrm{s}$, respectively. Before each rheological test, the sample was rejuvenated by a dynamic time sweep with $1000 \%$ strain at $0.1 \mathrm{rad} / \mathrm{s}$ for $600 \mathrm{~s}$ and then left to rest for $120 \mathrm{~s}$. A solvent trap was used to create a closed saturated atmosphere around the sample and thus minimize evaporation. In addition, for longer measurements (typically 1 day long), we have also used a ring of silicone oil, immiscible with water, sealing off the sample, and eliminating evaporation throughout the duration of the experiments.

\section{LAOS ANALYSIS}

In the linear regime, the Lissajous loop (stress versus strain within a period of oscillation) forms an ellipse where the major axis corresponds to the complex modulus, $G^{*}$, and the area of the loop is proportional to viscous modulus, $G^{\prime \prime}$. With increasing strain amplitude in the nonlinear regime, the stress response is no longer sinusoidal leading to a distortion of the Lissajous curves. The stress distortion was analyzed both by Fouriertransform analysis as well as the method of stress decomposition in an orthogonal set of Chebyshev polynomials [Ewoldt et al. (2008)]. The former determines the amplitude and phase difference of higher harmonics relative to the fundamental [Wilhelm et al. (1998)], while the latter uses symmetry arguments to decompose the total nonlinear stress response into a superposition of an elastic stress, $\sigma^{\prime}(x)$, where $x=\gamma / \gamma_{0}=\sin \omega t$, and a viscous stress, $\sigma^{\prime \prime}(y)$, where $y=\dot{\gamma} / \dot{\gamma}_{0}=\cos \omega t$. The total oscillatory stress is the sum of the two contributions, $\sigma(t)=\sigma^{\prime}(t)+\sigma^{\prime \prime}(t)=\gamma_{0} \sum_{n \text { :odd }}\left(G_{n}^{\prime} \sin n \omega t+G_{n}^{\prime \prime} \cos n \omega t\right)$. This decomposition is based on the idea that $\sigma^{\prime}$ should be an odd function of x and even of y, whereas $\sigma^{\prime \prime}$ should be even in $\mathrm{x}$ and odd in y. Such procedure results in single-valued $\sigma^{\prime}$ and $\sigma^{\prime \prime}$ functions of strain and strain rate, respectively, in contrast to the closed loops (see Fig. 6) obtained with the total stress versus strain (elastic Lissajous representation) and rate (viscous Lissajous representation). The elastic and viscous stresses may be directly related with the higher harmonics of a FT analysis [Cho et al. (2005); Ewoldt et al. (2008)]. The intracycle elastic and viscous nonlinearities may be quantified with the use of a set of orthogonal polynomials for describing $\sigma^{\prime}(t)$ and $\sigma^{\prime \prime}(t)$. Out of many, Chebyshev polynomials of the first kind were chosen [Ewoldt et al. (2008)] as the most appropriate, in terms of symmetry constrictions, that can also be easily related to the FT coefficients. Using this basis set, elastic and the viscous contributions to the stress can be written as

$$
\begin{aligned}
& \sigma^{\prime}(x)=\gamma_{0} \sum_{n: \text { odd }} e_{n}\left(\omega, \gamma_{0}\right) T_{n}(x), \\
& \sigma^{\prime \prime}(x)=\dot{\gamma}_{0} \sum_{n: \text { odd }} v_{n}\left(\omega, \gamma_{0}\right) T_{n}(y),
\end{aligned}
$$

where $T_{n}(x)$ and $T_{n}(y)$ are the nth-order Chebyshev polynomial of the first kind with $e_{n}\left(\omega, \gamma_{0}\right)$ and $v_{n}\left(\omega, \gamma_{0}\right)$, the corresponding amplitudes [Ewoldt et al. (2008)].

In the linear regime, only the first harmonic contributes to $\sigma^{\prime}(t)$ and $\sigma^{\prime \prime}(t)$; hence Eq. 1 recovers the linear viscoelastic results with $e_{1}=G^{\prime}$ and $v_{1}=G^{\prime \prime} / \omega=\eta^{\prime}$. In this limit, the 
elastic and viscous stresses are represented in the Lissajous plot by straight lines [see Figs. 6(a) and 6(b)]. When higher order Chebyshev coefficients are nonzero, the elastic and/or viscous stress contributions $\sigma^{\prime}(t)$ and $\sigma^{\prime \prime}(t)$ plotted against strain and rate, respectively, deviate from straight lines. In the nonlinear regime, a positive contribution of the third order polynomial results in an increased elastic stress at the maximum strain, $x$ $\rightarrow 1$, as compared to the first-order contribution alone. Thus, $e_{3}>0$ corresponds to an intracycle strain hardening, while $e_{3}<0$, resulting from a lower $\sigma^{\prime}(x)$ at $x \rightarrow 1$, indicates an intracycle strain softening. Similarly, for $v_{3}>0$, an increased (compared to a pure first-order contribution) viscous stress at $y \rightarrow 1$ corresponds to an intracycle shear thickening, whereas $v_{3}<0$ reflects an intracycle shear thinning. Equivalent information can be deduced from the minimum-strain amplitude elastic modulus, $G_{M}^{\prime} \equiv d \sigma /\left.d \gamma\right|_{x=0}=e_{1}-3 e_{3}$ $+\cdots$, and the large-strain elastic modulus, $G_{L}^{\prime} \equiv \sigma /\left.\gamma\right|_{x=1}=e_{1}+e_{3}+\cdots$, or their viscous counterparts which similarly are linear superpositions of $v_{n}$ [Ewoldt et al. (2008)].

In this way, the great advantage of such orthogonal decomposition compared to FT analysis is that the Chebyshev coefficients provide a clear physical classification of nonlinearities at large-strain amplitudes.

\section{RESULTS AND DISCUSSION}

Star-like micelles are soft particles with long polymeric arms which can strongly interpenetrate at high concentration reaching values much larger than overlap, $c^{*}$. Figure 1(a) shows the intensity auto-correlation function, $g_{2}(t)$, for a solution with $54.25 \mathrm{~g} / \mathrm{l}$ measured by MSDLS at $\theta=90^{\circ}$ at 15 and $20^{\circ} \mathrm{C}\left(2.5 c^{*}\right)$. An ultraslow relaxation of multimode character is observed in both cases. The elucidation of the origin of the ultraslow relaxation requires detailed q-dependent study which is beyond the scope of the present work and will be performed in the future. Briefly we note that the two steps observed [Fig. 1(a)] probably reflect heterogeneous slow dynamics similar to those observed in concentrated colloidal systems [El Masri et al. (2009)]. The observation that the ultraslow relaxation slows down strongly from 20 to $15{ }^{\circ} \mathrm{C}$ is naturally attributed to the increasing $R_{H}$ of the micelles with decreasing temperature leading to higher effective volume fraction and stronger arm interpenetration. Note that a $g_{2}(t=0)<1$ indicates the existence of a process with characteristic relaxation faster than $0.1 \mathrm{~s}$ due to the arm cooperative relaxation [Seghrouchni et al. (1998); Loppinet et al. (2005)].

The temperature dependence of $R_{H}$ affects also the linear viscoelastic properties of the system, as shown in Fig. 1(b). At $10{ }^{\circ} \mathrm{C}$, corresponding to an effective concentration $c$ $=2.64 c^{*}$ [as calculated from $\left.R_{H}(T)\right], G^{\prime}$ is larger than $G^{\prime \prime}$ in the whole frequency range and both moduli are almost frequency independent a typical feature of colloidal glasses [Mason et al. (1997); Stiakakis et al. (2002); Koumakis et al. (2008); Carrier and Petekidis (2009)]. At $20^{\circ} \mathrm{C}$ while $G^{\prime}>G^{\prime \prime}$ at high frequencies, it decreases with decreasing frequency until a terminal relaxation time is reached (about $0.4 \mathrm{rad} / \mathrm{s}$ ) below which $G^{\prime \prime}$ $>G^{\prime}$. At $25{ }^{\circ} \mathrm{C}\left(c=2.42 c^{*}\right)$ the system flows, however, not exhibiting a clear Maxwelllike $\left(G^{\prime} \propto \omega^{2}\right.$ and $\left.G^{\prime \prime} \propto \omega^{1}\right)$ behavior reminiscent of approaching a critical gelation [Winter and Mours (1997)]. For the solution with 54.25 g/l, Fig. 1(b) indicates a liquid-solid transition between 10 and $20{ }^{\circ} \mathrm{C}$, while a temperature sweep at $0.1 \mathrm{rad} / \mathrm{s}$ determines the transition at $\mathrm{T}_{c}=17{ }^{\circ} \mathrm{C}$. Here, most of the measurements are performed at $10{ }^{\circ} \mathrm{C}$. Figures 2(a) and 2(b) show the dynamic strain sweeps from the $54.25 \mathrm{~g} / \mathrm{l}$ solution $\left(c=2.5 c^{*}\right.$ at $\mathrm{T}=20^{\circ} \mathrm{C}$ ) at different frequencies [Fig. 2(a)] and temperatures [Fig. 2(b)]. In all cases, the samples display the typical shear melting transition for colloidal glasses [Mason et al. (1997); Pham et al. (2006); Carrier and Petekidis (2009)]. At low strains, the sample exhibits a solid-like behavior; then as the strain amplitude is increased, the sample starts 

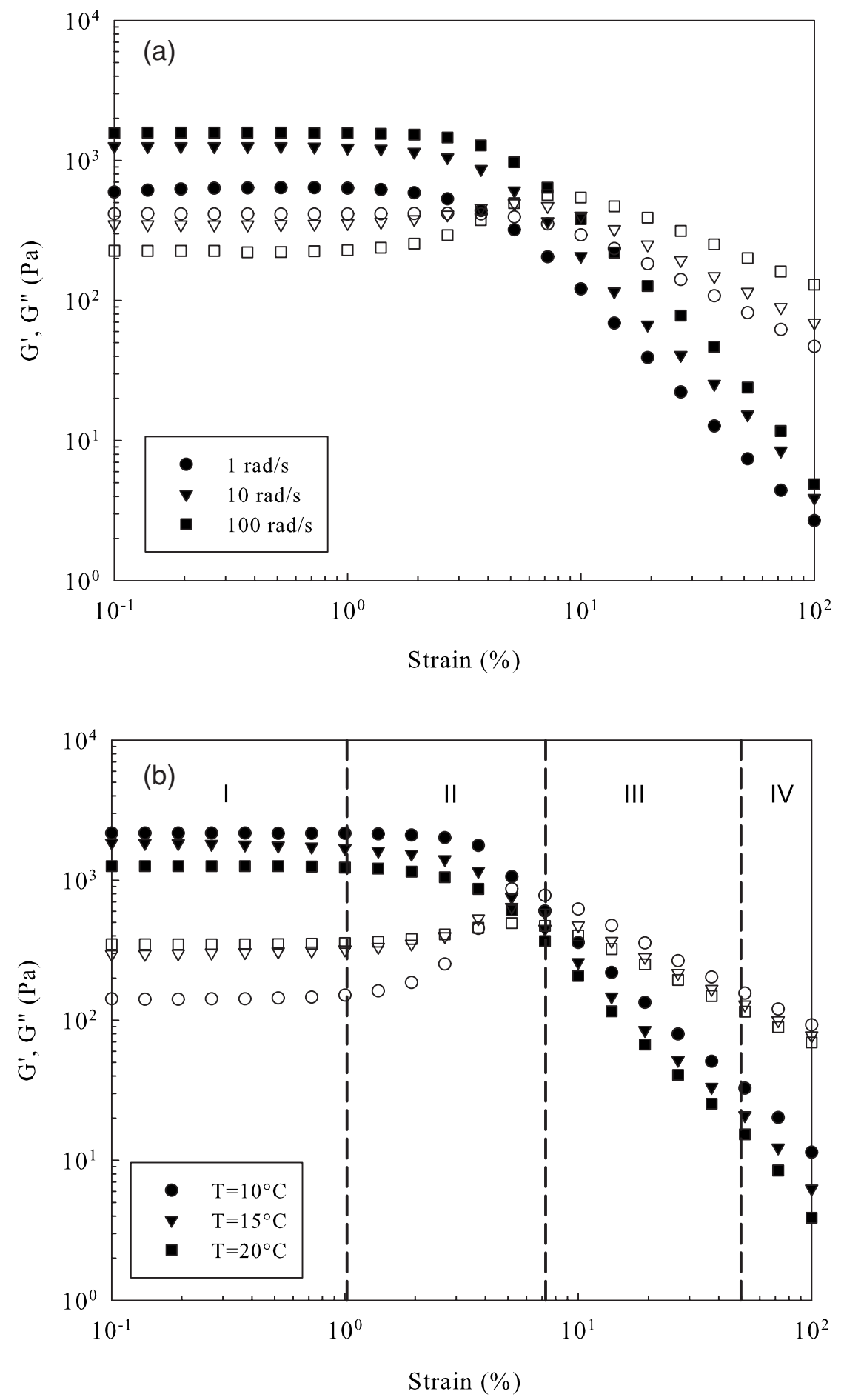

FIG. 2. (a) Dynamic strain sweeps for a solution of PEP-PEO with $54.25 \mathrm{~g} / \mathrm{l}$ versus frequency at $20{ }^{\circ} \mathrm{C}(c$ $\left.=2.5 c^{*}\right) . G^{\prime}$ are indicated with closed and $G^{\prime \prime}$ with open symbols. (b) Dynamic strain sweeps for a solution of PEP-PEO with $54.25 \mathrm{~g} / 1$ versus temperature at $\omega=10 \mathrm{rad} / \mathrm{s}$ as indicated: $\mathrm{T}=10{ }^{\circ} \mathrm{C}\left(c=2.64 c^{*}\right), \mathrm{T}=15{ }^{\circ} \mathrm{C}(c$ $\left.=2.57 c^{*}\right), \mathrm{T}=20^{\circ} \mathrm{C}\left(c=2.5 c^{*}\right) . G^{\prime}$ are indicated with closed and $G^{\prime \prime}$ with open symbols. Regions from I to IV are discussed in the text. 


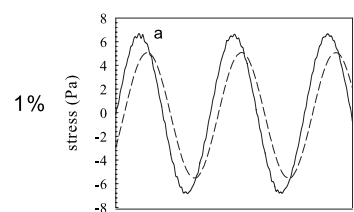

time

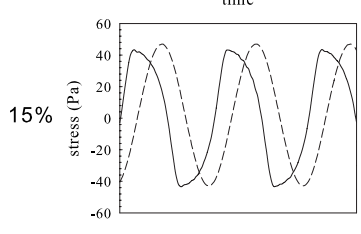

time

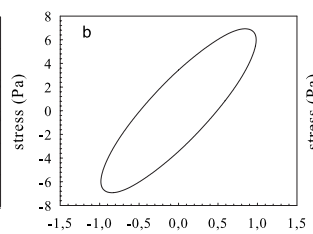

Strain $(\%)$

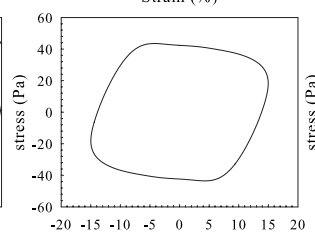

Strain (\%)

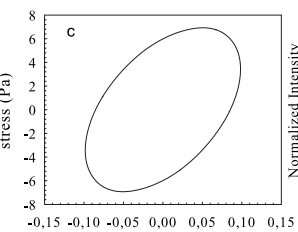

rate $\left(\mathrm{s}^{-1}\right)$

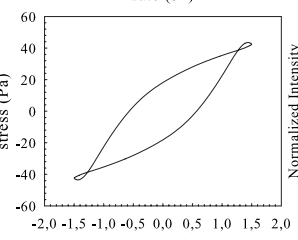

rate $\left(\mathrm{s}^{-1}\right)$
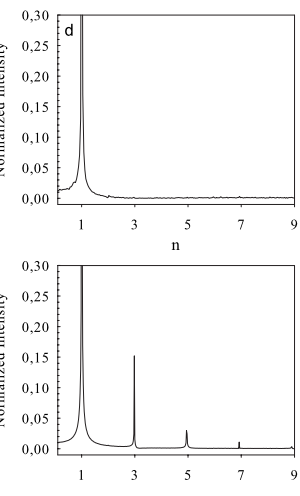

$\mathrm{n}$

FIG. 3. (a) Shape of the stress response (full lines) and strain (dotted lines) as a function of time, (b) Lissajous plot of the stress as a function of strain (elastic representation) and (c) strain rates (viscous representation), and (d) Fourier spectra. The data were collected by LAOS experiments from a solution of PEP-PEO with 54.25 g/l and $\mathrm{T}=10{ }^{\circ} \mathrm{C}\left(c=2.64 c^{*}\right)$ for $\gamma_{0}=1 \%$ (top panel) and $\gamma_{0}=15 \%$ (bottom panel).

yielding with $G^{\prime}$ decreasing while $G^{\prime \prime}$ first passes through a maximum before decreasing as well. Above the overlap of $G^{\prime}$ and $G^{\prime \prime}$, which almost coincides with the peak of $G^{\prime \prime}$, both moduli decrease with $G^{\prime \prime}>G^{\prime}$ indicative of a shear melted sample. The yield strain $\left(\gamma_{y}\right)$, determined at the $G^{\prime}-G^{\prime \prime}$ crossover (or at the maximum of $G^{\prime \prime}$ ), increases weakly (from 5 to 10\%) with increasing frequency in accordance with previous findings in hard and soft spheres [Petekidis et al. (2003); Helgeson et al. (2007); Le Grand and Petekidis (2008); Derec et al. (2003); Pham et al. (2008)].

The temperature dependence for $54.25 \mathrm{~g} / 1\left(c=2.5 c^{*}\right.$ at $\left.\mathrm{T}=20^{\circ} \mathrm{C}\right)$ [Fig. 2(b)] shows an increasing $G^{\prime}$ and a decreasing $G^{\prime \prime}$ with decreasing temperature due to the accompanied effective volume fraction increase and the resulting stronger arm interpenetration. On the other hand, $\gamma_{y}$ remains virtually the same due to the constant number concentration and thus the unchanged distance between centers of particles that determine the size of the cage. Such behavior also suggests that decreasing temperature is not quantitatively equivalent to increasing number concentration. Thus, decreasing temperature leads to particle swelling but does not affect the aggregation number, which means that the particles become bigger but their repulsive interaction become weaker in agreement with recent experimental findings in other soft particles [Le Grand and Petekidis (2008); Renou et al. (2009); van Ruymbeke et al. (2010)]. The strain regimes (I-IV) denoted in Fig. 2(b) indicates different mechanical responses discussed below.

\section{FT-ANALYSIS}

Dynamic strain sweeps monitor the average viscoelastic properties over few periods of oscillation, whereas the stress response within a cycle may provide more detailed information especially in the non-linear regime. Figure 3 shows the stress response as a function of time (panel a), applied strain (panel b), and strain rate (panel c) within a period of oscillation for two applied strain amplitudes. The upper part of Fig. 3 shows the response under $1 \%$ strain (linear regime) where the stress remains sinusoidal and no distortion is observed. In this case, the elastic Lissajous plot [Fig. 3(b)] is an ellipsoid where the slope of the main axis corresponds to $G^{*}$, the tangent slope at zero strain yields the linear elastic modulus $G^{\prime}$, and the area is proportional to $G^{\prime \prime}$. The non-deformed ellipsoidal shape is the signature of the linear regime. The linear regime is clearly con- 
firmed by the FT analysis [see Fig. 3(d)] which shows only a contribution from the fundamental frequency in the stress response. On the other hand, the lower part of Fig. 3 shows the stress response for applied strain amplitude of $15 \%$, i.e., in the non-linear regime. The stress signal is strongly deformed producing a much more open, square-like Lissajous loop in the elastic representation. The non-linearity can be quantified through the FT analysis which reveals strong contribution from higher odd harmonics, as seen in Fig. 3(d) (bottom). Note that even harmonics were virtually zero suggesting the absence of transient wall slip [Graham (1995); Wilhelm et al. (1998)] or secondary flows [Atalık and Keunings (2004)] that may break the symmetry of the Lissajous curves. Such symmetry also (see also Figs. 6 and 9 below) indicates that steady state was reached and no preferred direction of flow exists in contrast with observations by Rogers and Vlassopoulos (2010) in multiarm stars.

A systematic FT analysis is shown in Fig. 4(a) where the higher harmonic contributions relative to the fundamental, $I_{n} / I_{1}$, are plotted versus the applied strain. In the linear regime, below $\gamma_{0}=2 \%$, only the fundamental frequency contributes to the stress response. At higher strains, the contribution of higher odd harmonics becomes more pronounced due to the increased stress nonlinearity. As the strain is increased, first, the third harmonics contribution sets in and then the fifth and seventh. $I_{3} / I_{1}$ increases with increasing strain amplitude up to about $\gamma_{0}=100 \%$ and then reaches a constant value of $I_{3} / I_{1}$ $\sim 24 \%$. The fifth and the seventh harmonics also increase with strain but are weaker than the third one while they do not seem to reach a plateau, at least in the strains accessible here. Moreover, for all frequencies studied, $I_{n} / I_{1}$ is always less than $1 / \mathrm{n}$ as expected for simple shear thinning models [Wilhelm et al. (2000)]. Note, however, that for more complicated nonlinear response, with strong elastic contribution such scaling does not necessarily hold. In addition, the scaling $I_{3} / I_{1} \propto \gamma_{0}^{2}$ proposed for the weakly nonlinear regime [Neidhöfer et al. (2003)] does not seem to describe accurately intermediate strains here; a scaling exponent of about 1.5 is found instead for strains between $2 \%$ and $10 \%$. We further observe that the onset of stress nonlinearity is where $G^{\prime \prime}$ starts to increase in the conventional dynamics strain sweep [Fig. 2(b)]. The FT analysis at different frequencies (from 1 to $20 \mathrm{rad} / \mathrm{s}$ ) is summarized in Fig. 4(b). Although $I_{3} / I_{1}$ exhibits essentially the same shape for all frequencies, the strain at which $I_{3} / I_{1}$ starts to increase shifts to higher strains with increasing frequency, consistently with the increase of the yield strain determined at the peak of $G^{\prime \prime}$ or the $G^{\prime}-G^{\prime \prime}$ crossover.

Figure 5(a) shows the viscoelastic data in a dynamic strain sweep at $10 \mathrm{rad} / \mathrm{s}$ together with values acquired at steady state during successive dynamic time sweeps at specific strain amplitudes. The latter where used for the measurement of the stress response within a cycle and the determination of the higher harmonics [Figs. 4 and 5(b)]. Figure 5 demonstrates that both measurements lead to almost identical values; hence the data from dynamic strain sweeps are steady state values.

In Fig. 5(b) we show the behavior of the third harmonic at different number concentrations (at $\mathrm{T}=10{ }^{\circ} \mathrm{C}$ ). Although the onset of the $I_{3} / I_{1}$ increase is observed at lower strains with increasing concentration, almost the same maximum value is reached in all samples. At higher number concentrations the star-like micelles interpenetrate each other more and their repulsive interactions increases [Roovers et al. (1995); Renou et al. (2007)] leading to higher viscoelastic moduli similarly with the response at constant number concentration and decreasing temperature [Fig. 2(b)]. However, the yield strain decreases with increasing number concentration (inset of Fig. 5) in contrast to what was observed with increasing effective volume fraction via decreasing temperature. This finding is indicative of the non-equivalence of $\mathrm{T}$ and $\mathrm{c}$ dependence of the effective volume fraction as mentioned earlier. When the data from different concentrations [Fig. 5(b)] are 

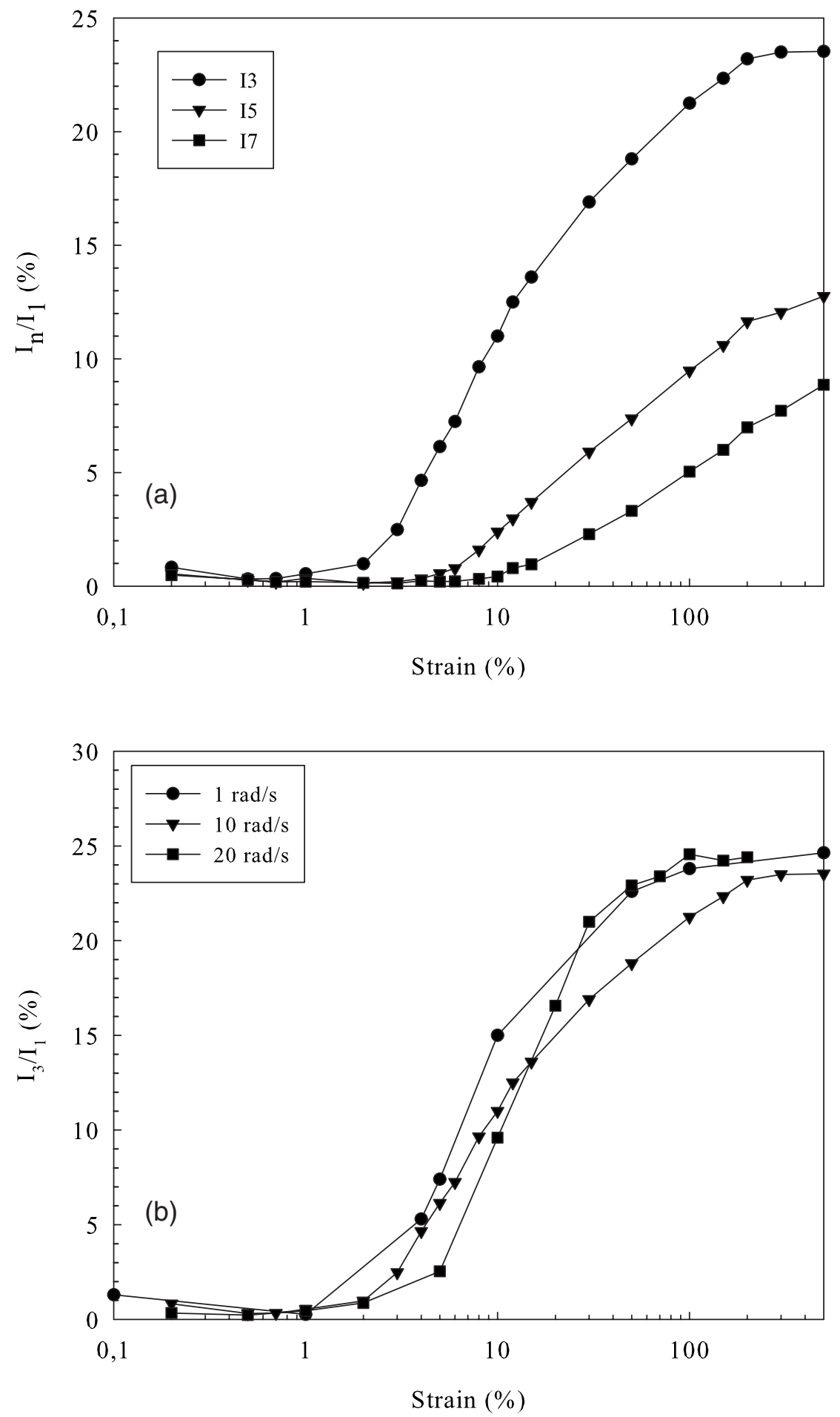

FIG. 4. Normalized amplitude $I_{n} / I_{1}$ for the third $(\bullet)$, the fifth $(\boldsymbol{\nabla})$ and the seventh $(\boldsymbol{\square})$ harmonics as a function of the applied strain for a solution of PEP-PEO for $54.25 \mathrm{~g} / 1$ at $\mathrm{T}=10{ }^{\circ} \mathrm{C}\left(c=2.64 c^{*}\right)$ and $\omega=10 \mathrm{rad} / \mathrm{s}$. (b) Normalized amplitude $I_{3} / I_{1}$ as a function of the applied strain for a solution of PEP-PEO for $54.25 \mathrm{~g} / \mathrm{l}$ at $\mathrm{T}$ $=10{ }^{\circ} \mathrm{C}\left(c=2.64 c^{*}\right)$ for different frequencies. 

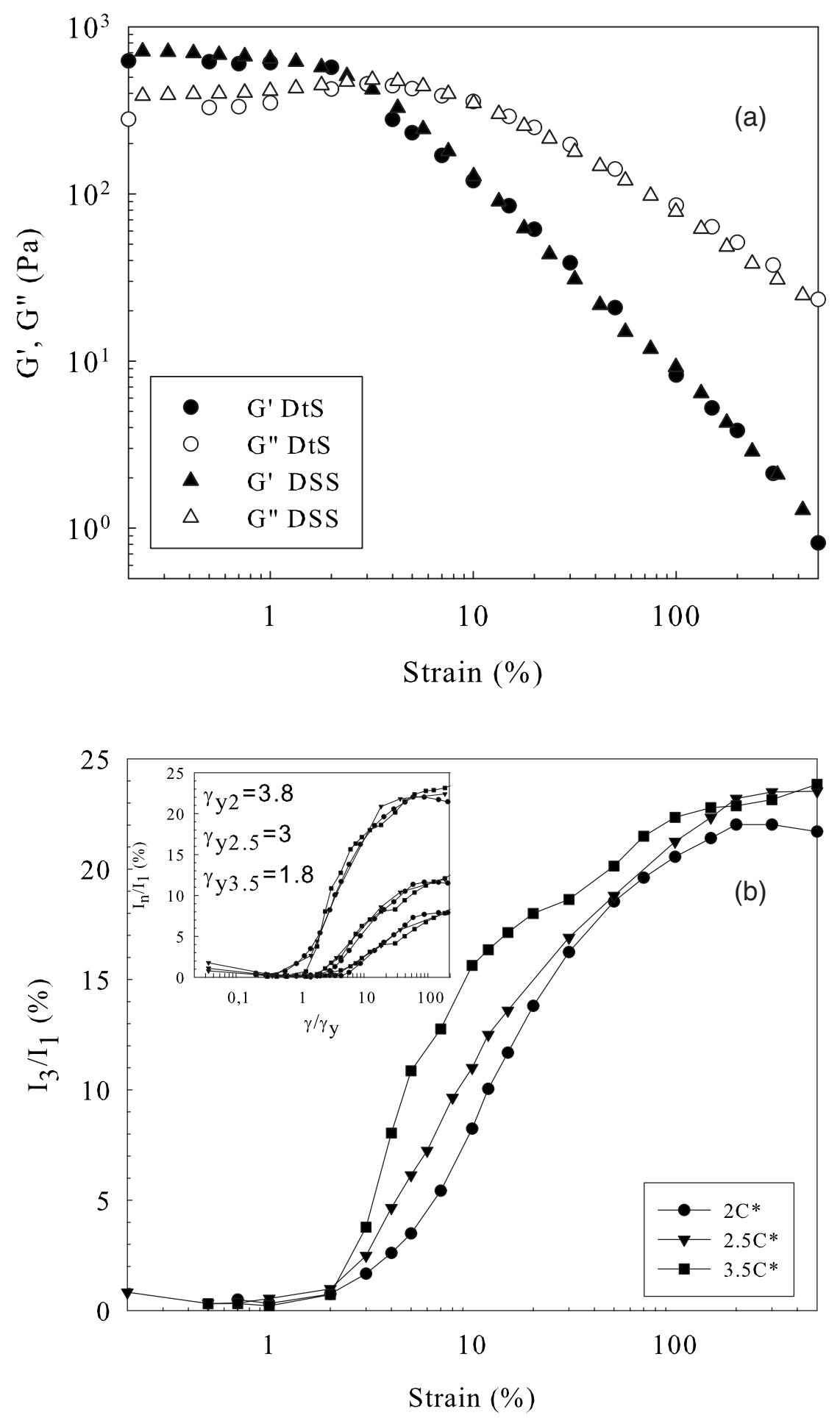

FIG. 5. (a) $G^{\prime}$ (filled symbols) and $G^{\prime \prime}$ (open symbols) as a function of strain amplitude from a dynamic strain sweep (triangles) and at steady state from time sweep at a specific strain (circles) for a solution of PEP-PEO at $\mathrm{T}=10{ }^{\circ} \mathrm{C}\left(c=2.64 c^{*}\right)$. (b) Normalized amplitude $I_{3} / I_{1}$ as a function of the applied strain at $\omega=10 \mathrm{rad} / \mathrm{s}$ for a solution of PEP-PEO at $\mathrm{T}=10{ }^{\circ} \mathrm{C}$ at different concentrations as indicated. Inset: $I_{n} / I_{1}$ as a function of the reduced strain, $\gamma / \gamma_{y}$, for the same concentrations. 
a
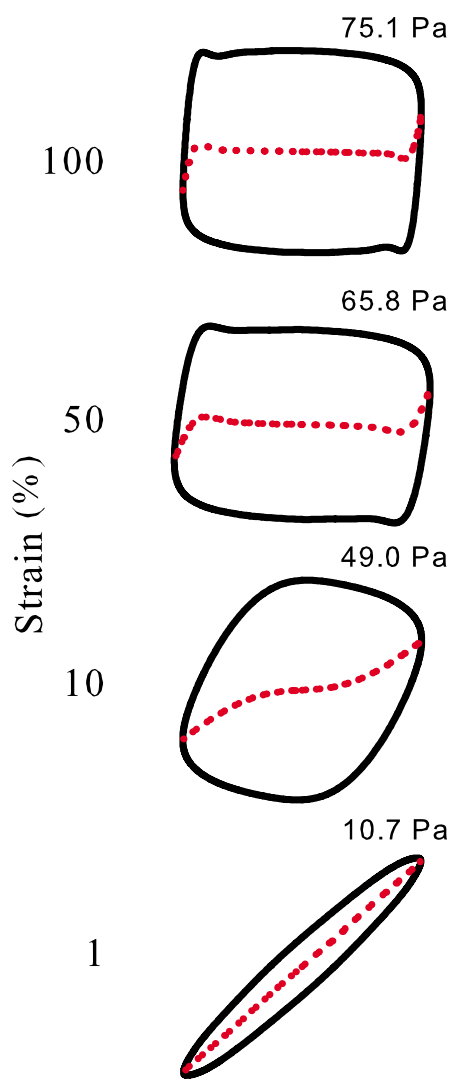

b
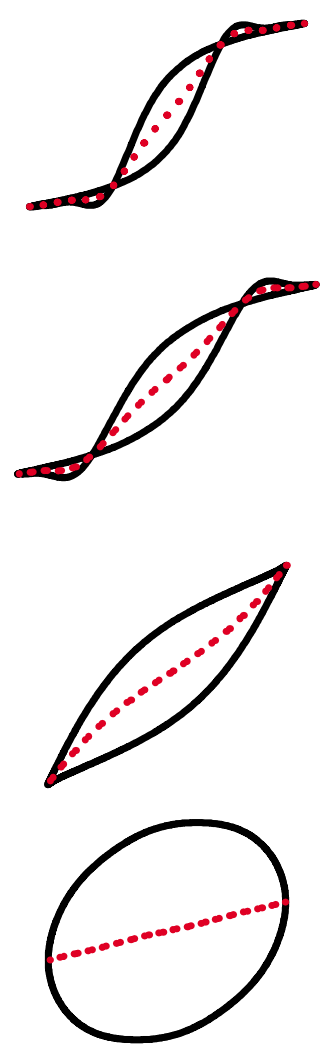

C
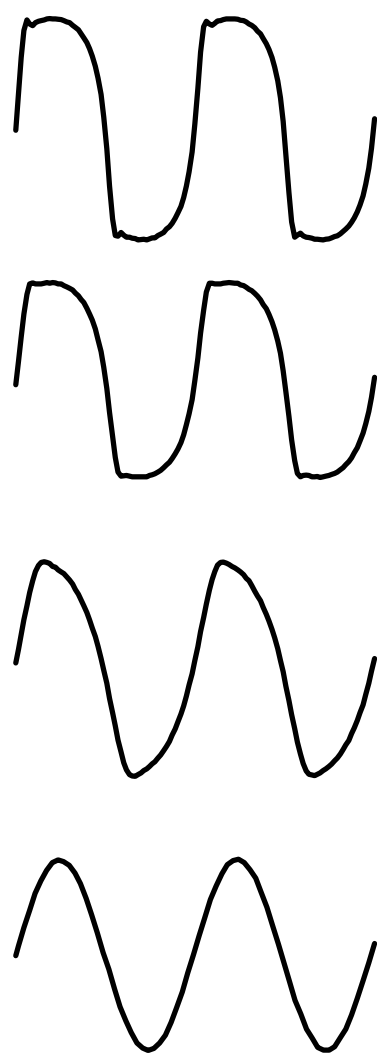

FIG. 6. (a) (left column) Elastic Lissajous curves map for different strains at $10 \mathrm{rad} / \mathrm{s}$ for a solution of PEP-PEO at $\mathrm{T}=10{ }^{\circ} \mathrm{C}\left(c=2.64 c^{*}\right)$. The solid lines are the normalized total stress $(\sigma)$ response and the dotted lines are the elastic stress response $\left(\sigma^{\prime}\right)$ as a function of the normalized strain $(\gamma)$. The maximum stresses are indicated above each curve. (b) (central column) Viscous Lissajous curves map corresponding to the same strains as in (a). The solid lines are the normalized total stress $(\sigma)$ response and the dotted lines are the viscous stress response $\left(\sigma^{\prime \prime}\right)$ as a function of the normalized strain rate $(\dot{\gamma})$. (c) (right column) The corresponding stress response within a cycle.

renormalized by the corresponding yield strain, they superimpose quite well for all higher harmonics [inset of Fig. 5(b)]. This demonstrates explicitly that the magnitude of the nonlinearities is determined by the distance from the yield strain and thus all concentrations in the regime studied here behave similarly.

\section{LISSAJOUS PLOTS}

Examples of Lissajous curves are presented in Fig. 6 for various imposed strain amplitudes $\left(\gamma_{0}\right)$ at a representative frequency of $\omega=10 \mathrm{rad} / \mathrm{s}$. Both the elastic $[\sigma(t)$ vs $\gamma(t)]$ and the viscous representation $[\sigma(t)$ versus $\dot{\gamma}(t)]$ are shown together with corresponding stress waveforms. At $1 \%$ strain, i.e., in the linear regime, the elastic Lissajous loop is an ellipse as expected and the elastic contribution to the stress (dotted line in left column of Fig. 6) is a straight line where the slope is equal to $G^{\prime}$ and its value is in accordance with the one obtained by the dynamic strain sweeps or the frequency sweeps in the linear 
regime. In the same way in the central column of Fig. 6, the dotted lines representing the intracycle viscous stress are also straight lines at low strain amplitudes with their slopes equal to $\eta^{\prime}=G^{\prime \prime} / \omega$. As the strain is increased, the onset of nonlinearity in the material response is evident by a visual inspection of distorted Lissajous loops, which appear more open in the elastic and self-intersecting in the viscous representations, respectively. At the same time, the concurrent elastic and viscous stresses progressively depart from linearity with increasing strain or rate revealing a rather rich material response with softening/ hardening and thinning/thickening contributions depending on the frequency as will be discussed below. An intriguing observation is that in the nonlinear regime the minimumstrain modulus, $G_{M}^{\prime}$, may even become negative due to strong nonlinear strain hardening contribution $\left(e_{1}<3 e_{3}\right)$. A similar finding was reported by Ewoldt et al. (2008) in wormlike micelle solutions, and will be discussed further below.

\section{CHEBYSHEV POLYNOMIAL ANALYSIS}

As mentioned above, the use of Chebyshev polynomials allows decomposition of the stress response into purely elastic and viscous components, represented by $e_{n}$ and $v_{n}$. Here we are focusing at $e_{3}$ and $v_{3}$ since they are significantly larger than higher order contributions in all cases. When the applied strain amplitude approaches or exceeds the yield strain the elastic and viscous stresses depart from straight lines indicating a significant contribution from, at least, the third order Chebyshev coefficients $\left(e_{3}\right.$ and $\left.v_{3}\right)$, as seen in Fig. 6. The results for the elastic and viscous responses at 1 and $10 \mathrm{rad} / \mathrm{s}$ are very similar. By decomposing the elastic and the viscous stress signal to the orthogonal set of Chebyshev polynomial, we may determine quantitatively the intracycle strain hardening/ softening and shear thinning/thickening response of the material.

Figure 7 depicts $e_{3} / e_{1}\left(=-G_{3}^{\prime} / G_{1}^{\prime}\right)$ and $v_{3} / v_{1}\left(=G_{3}^{\prime \prime} / G_{1}^{\prime \prime}\right)$ as a function of the applied strain at $10 \mathrm{rad} / \mathrm{s}$. In the linear regime, no contribution from higher order intracycle elastic or viscous stresses is detected. With increasing strain above about 3\% (coinciding with the increase of $G^{\prime \prime}$ in the strain sweep), $v_{3} / v_{1}$ starts to increase revealing a shear thickening contribution while no elastic contribution in the nonlinearity is recorded (see arrow 1 in Fig. 7). This suggests that for these strains the nonlinearity has a purely viscous origin. The fact that it is accompanied by an increase of the total $G^{\prime \prime}$ suggests a regime of increased energy dissipation and thus viscosity of both linear and non-linear character. Increasing the strain amplitude above $5 \%-6 \% e_{3} / e_{1}$ starts to increase indicating an additional strain hardening non-linear contribution while at the same time $v_{3} / v_{1}$ drops and becomes negative above about $15 \%$ strain (see arrow 2 in Fig. 7). At this particular strain regime $(5 \%$ to $15 \%)$, the sample exhibits nonlinearities in both the elastic and viscous responses attributed to strain hardening and shear thickening, respectively. Above $15 \%$ strain, $v_{3}$ becomes negative, revealing a shear thinning nonlinear viscous response, while $e_{3}$ keeps increasing strongly probing a pronounced strain hardening type of nonlinear elastic behavior.

At even higher strains, both the elastic and viscous contributions of the third order Chebyshev coefficients seems to reach a plateau similarly with the higher harmonics from the FT analysis [Figs. 4 and 5(b)]. Note that the latter relate to Chebyshev coefficients through $I_{3} / I_{1}=\sqrt{e_{3}^{2}+\left(v_{3} \omega\right)^{2}} / \sqrt{e_{1}^{2}+\left(v_{1} \omega\right)^{2}}$ [Ewoldt et al. (2008)]. Hence $I_{3} / I_{1}$ is in general less sensitive to intracycle non-linearities than $e_{3} / e_{1}$ and $v_{3} / v_{1}$ since a strong elastic or viscous non-linearity represented by the latter may be covered in the former by a strong linear contribution. The elastic stress exhibits a maximum strain hardening response with $G_{3}^{\prime}$ reaching about $1.2 G_{1}^{\prime}$ while the viscous stress shows a shear thinning contribution with $\eta_{3}^{\prime}=-0.3 \eta_{1}^{\prime}$. However, since in this regime (region IV in the inset of Fig. 7) $G^{\prime \prime}$ is 


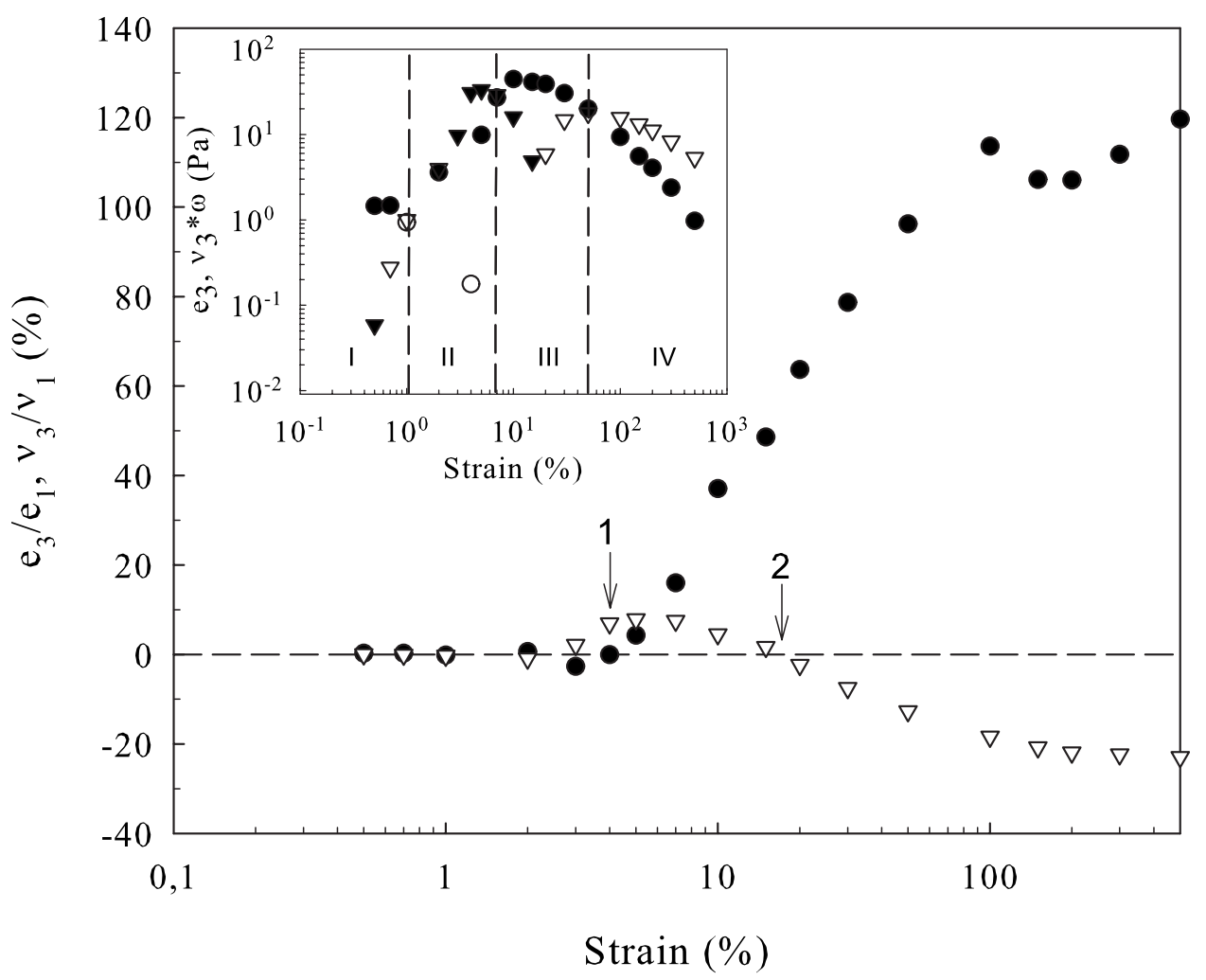

FIG. 7. Relative third order elastic, $e_{3} / e_{1}$, (black circles) and viscous, $v_{3} / v_{1}$ (open triangles) Chebyshev coefficients as a function of $\gamma_{0}$ at $\omega=10 \mathrm{rad} / \mathrm{s}$ for a solution of PEP-PEO at T=10 ${ }^{\circ} \mathrm{C}\left(c=2.64 c^{*}\right)$. The inset represents the absolute values of the strain hardening $\left(e_{3}, \bullet\right)$, strain softening $\left(-e_{3}, \bigcirc\right)$, shear thickening $\left(v_{3} \omega, \boldsymbol{\nabla}\right)$, and the shear thinning $\left(-v_{3} \omega, \nabla\right)$ contribution as a function of $\gamma_{0}$. The regions from I to IV are described in the text.

larger than $G^{\prime}$, one has to consider the absolute values of $e_{3}$ and $v_{3}$ in order to determine the dominant strain hardening/shear thinning contributions of the third harmonics. The absolute values of $e_{3}$ and $v_{3} \omega$ are shown in the inset of Fig. 7 in a log-log representation where the negative values of $e_{3}$ and $v_{3}$ (i.e. strain softening and shear thinning respectively) are also indicated (open symbols). At high strains, $-v_{3} \omega$ is much larger than $e_{3}$ suggesting that the effective shear thinning contribution is much stronger than the strain hardening one. This representation clearly shows the smooth transition from shear thickening to strain hardening to shear thinning contribution of the third harmonics with increasing strain (from region II to region IV). Note, however, that these characterizations signify only the predominant contribution in intracycle nonlinearities. The individual elastic and viscous contributions (for example, strain hardening and shear thinning, respectively) coexist with each other showing a complicated yielding response. As $\gamma_{0}$ increases (in the regime where $G^{\prime \prime}>G^{\prime}$ ), the relative amplitude between the elastic and viscous contributions decreases. Thus, at a strain of about $70 \%$, the intra-cycle strain hardening response $\left(e_{3}\right)$ is equal to the intra-cycle shear thinning response $\left(v_{3} \omega\right)$ indicating a transition from an elastic-modulated viscous response to a viscous modulated viscous response. This means that while the average (over the period), mechanical response is that of a viscous shear melted glass with $G^{\prime \prime}>G^{\prime}$, since the strain amplitude exceeds the yield strain, the nonlinearities within the oscillation cycle indicate a temporary in- 


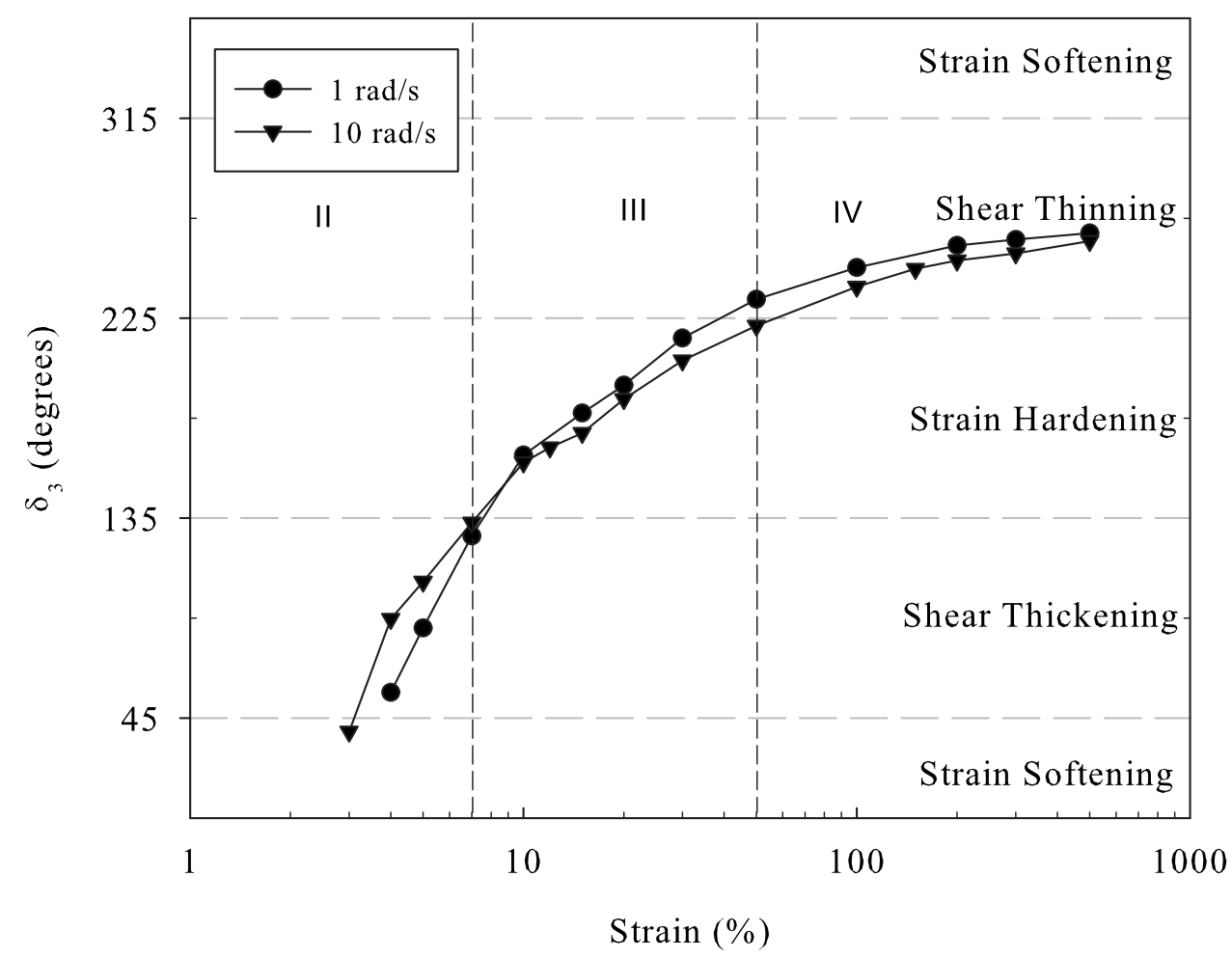

FIG. 8. Phase of the third harmonics $\left[\delta_{3}=\arctan \left(-v_{3} \times \omega / e_{3}\right)\right]$ as a function of $\gamma_{0}$ for a solution of PEP-PEO at $\mathrm{T}=10{ }^{\circ} \mathrm{C}\left(c=2.64 c^{*}\right)$ at 1 and $10 \mathrm{rad} / \mathrm{s}$. The dash lines delimit the different strain hardening/softening shear thickening/thinning dominant regions.

crease of the elasticity (strain hardening) and finally at the highest strains reached a temporary decrease of the viscosity below its average values (shear thinning).

Another illustration of the intracycle response is represented in Fig. 8 where the phase difference of the third harmonics $\left[\delta_{3}=\arctan \left(-v_{3} \times \omega / e_{3}\right)\right]$ is plotted versus the strain amplitude for two of the frequencies investigated. It should be noted that $\delta_{3}$ differs from the phase difference between the third harmonic and the fundamental which has been commonly used in other studies [Neidhöfer et al. (2003); Le Grand and Petekidis (2008)]. Similar to $\tan \delta\left(=G^{\prime \prime} / G^{\prime}\right)$ representing in the linear regime, the ratio of the viscous and the elastic contribution in the average mechanical response, $\delta_{3}$, denotes the relative strength of the viscous $\left(v_{3} \omega\right)$ to the elastic $\left(e_{3}\right)$ nonlinearity within an oscillation cycle. Thus the range where $-e_{3}$ is greater than $\left|v_{3}\right| \omega$ corresponds to a strain softening dominant contribution; this corresponds to $-45^{\circ}<\delta_{3}<45^{\circ}$. Similarly, when $\left|v_{3}\right| \omega$ is larger, the dominant mechanism is that of shear thickening with $45^{\circ}<\delta_{3}<135^{\circ}$. The same reasoning is applied to determine the strain hardening and shear thinning dominated regimes where $135^{\circ}<\delta_{3}<225^{\circ}$ and $225^{\circ}<\delta_{3}<-315^{\circ}$, respectively. As seen in Fig. 8, the results at 1 and $10 \mathrm{rad} / \mathrm{s}$ are very similar as was already obvious from the Lissajous curves. In both frequencies, we observe a smooth transition from a shear thickening dominant non-linear response to a strain hardening and finally a shear thinning dominant one. The initial intracycle shear thickening behavior of the third harmonics coincides with the increase of $G^{\prime \prime}$ in the dynamic strain sweep. Such average and intracycle increase in energy dissipation maybe related with additional, to the Brownian contribution: shear 
induced in-cage diffusion. Then as the strain amplitude get higher the sample yields and both $G^{\prime}$ and $G^{\prime \prime}$ start to drop leading eventually to a $G^{\prime \prime}$ much bigger than $G^{\prime}$. Nevertheless, the intracycle analysis following the third order Chebyshev coefficient reveals transitions from a strain hardening to a shear thinning mechanisms, while the conventional average rheological response shows only a generic shear melting behavior. The intracycle strain hardening reflects increase of the maximum strain elasticity of the system for strain amplitudes around the yield strain. On the other hand, the shear thinning intracycle behavior observed at higher strains might be related to pronounced post-yield out-of-cage shear induced particle rearrangements [Petekidis et al. (2002)]. This succession of events points out that the yielding and flow in such systems is a combination of different nonlinear intracycle physical mechanisms

\section{FREQUENCY DEPENDENCE/RELATION WITH STEADY SHEAR}

As seen in Figs. 6 and 8, the nonlinear behavior seems similar at 1 and $10 \mathrm{rad} / \mathrm{s}$. In this section, we look in more detail the frequency dependence of the stress response in the post-yield regime at a constant strain amplitude. Figure 9 shows the elastic and the viscous Lissajous curves for different frequencies (from 0.5 to $20 \mathrm{rad} / \mathrm{s}$ ) at $\gamma_{0}=100 \%$ together with the corresponding stress curves. The total stress exhibits a clear stress overshoot at the beginning and reversing of a strain cycle, which is then followed by a broad bump and a final decrease at the very end of the half period. As the frequency is increased, the first stress overshoot becomes a bit more pronounced and finally at the highest frequency of $20 \mathrm{rad} / \mathrm{s}$, a second peak or oscillation in the stress response (right column of Fig. 9) is observed. Such feature is also reflected in both the elastic and viscous Lissajous representations (left and central columns of Fig. 9). The study of the frequency dependence of the elastic and viscous contribution to the stress may add further insight in the understanding of physical mechanisms involved in nonlinear response at different strain regimes, as observed in Figs. 6 and 8. Unfortunately, however, the determination of intracycle stress distortion and the quantitative analysis of higher harmonics at frequencies larger than $20 \mathrm{rad} / \mathrm{s}$ are significantly affected by instrumental effects of the ARES strain-controlled rheometer related to the natural frequency of the transducer. Nonlinearities at higher frequencies are under investigation and will be presented elsewhere [Poulos et al. (2010)].

A variety of colloidal glasses and concentrated suspensions exhibit stress overshoots during a steady step rate experiments around a strain of $10 \%$, which is attributed to cage breaking [Gadala-Maria and Acrivos (1980); Liddel and Boger (1996); Derec et al. (2003); Stokes and Telfor (2004); Carrier and Petekidis (2009)]. Such overshoot in a step rate experiment at $\dot{\gamma}=0.5 \mathrm{~s}^{-1}$ is shown in Fig. 10 (inset) for the $54.25 \mathrm{~g} / \mathrm{l}\left(c=2.68 c^{*}\right.$ at $\mathrm{T}=10^{\circ} \mathrm{C}$ ) together with the oscillatory stress response within one period. The behavior in oscillatory and steady shear may be viewed as the result of the same underlying phenomenon, i.e., shear induced particle rearrangements at the microscopic to mesoscopic level of the cage. The initial stress increase corresponds to the elastic deformation of the cage and the build up of stress until the cage breaks dissipating the stored stress through irreversible particle rearrangements (gray region in the inset of Fig. 10). This relaxation is followed by a stress plateau corresponding to a steady state viscous flow of the sample at constant strain rate. In a LAOS experiment, the total stress response in the positive half period of the stress (Fig. 10) looks very similar to the stress response during a step rate experiment (inset of Fig. 10) even though the rate during this part of the oscillation is not constant. This comparison allows us to qualitatively relate the steady rate measurements to the nonlinear oscillatory experiments where the elastic and viscous 
a
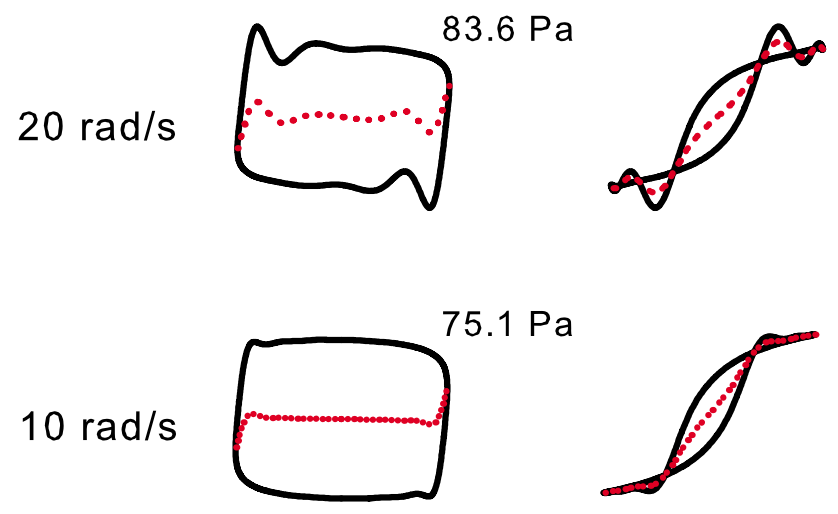

b

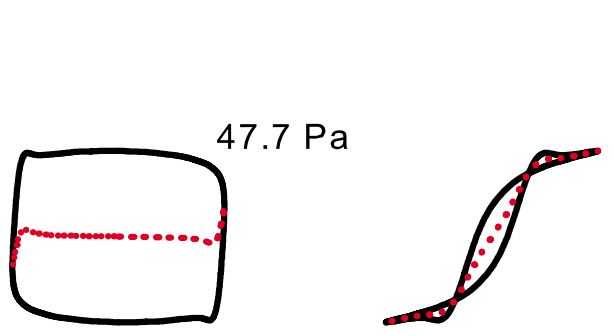

37.7 $\mathrm{Pa}$

\section{$0,5 \mathrm{rad} / \mathrm{s}$}
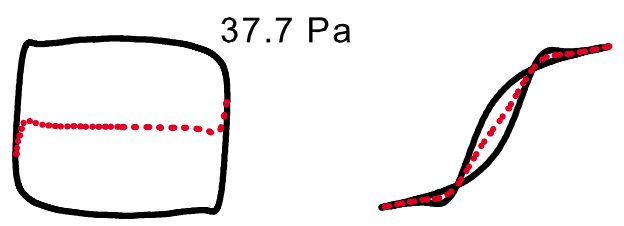
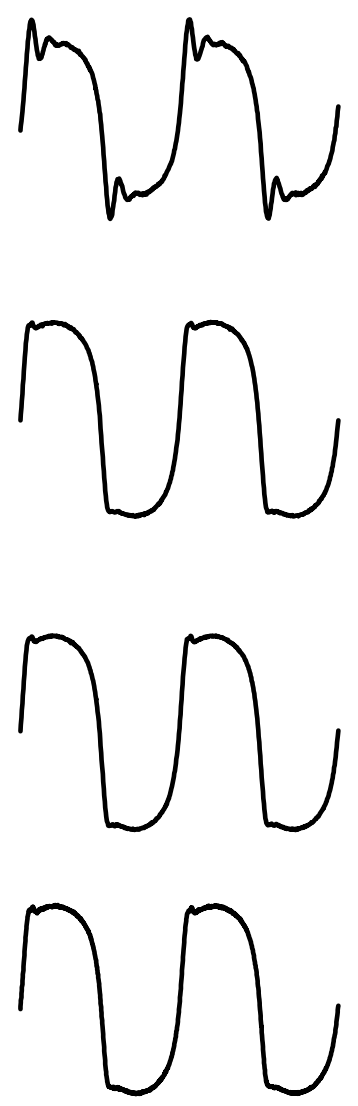

FIG. 9. Elastic (a) and viscous (b) Lissajous curves map for different frequencies at $\gamma_{0}=100 \%$ for a solution of PEP-PEO at $\mathrm{T}=10{ }^{\circ} \mathrm{C}\left(c=2.64 c^{*}\right)$. The solid lines are the normalized total stress $(\sigma)$ response and the dotted lines are the elastic stress response $\left(\sigma^{\prime}\right)$ (a) as a function of the normalized strain $(\gamma)$ and the viscous stress response $\left(\sigma^{\prime \prime}\right)(\mathrm{b})$ as a function of the normalized strain rate $(\dot{\gamma})$. The maximum stresses are indicated above each curve. The corresponding shape of the stress response as a function of time is shown in panel $\mathrm{c}$.

dominated regions over half a period may be determined (Fig. 10), in accordance with what has been previously suggested in the literature [for example, Gadala-Maria and Acrivos (1980); Carrier and Petekidis (2009); Rogers and Vlassopoulos (2010)]. We see that, in analogy to steady shear, when the strain amplitude in an oscillatory experiment exceeds the yield strain, initially the stress increases elastically due to cage deformation that results in a stress overshoot at the yield point where the cage starts breaking. This is followed by a stress relaxation and subsequently by viscous flow of the shear melted glass. Then at the middle of half the period (or at maximum rate) and beyond, when the strain still increases, the stress starts to drop due to cage reformation under weak and then reverse shear rate (Fig. 10). Thus, for low frequencies the total stress response is predominantly viscous. This explains the narrow first stress peak followed by a broad bump in the stress response as a function of time [Fig. 9(c)] or strain in the elastic Lissajous representation [Fig. 9(a)] from 0.5 to $10 \mathrm{rad} / \mathrm{s}$. Similarly in step rate tests, the stress overshoot is weakening with decreasing rate (or Péclet number) due to the increased 


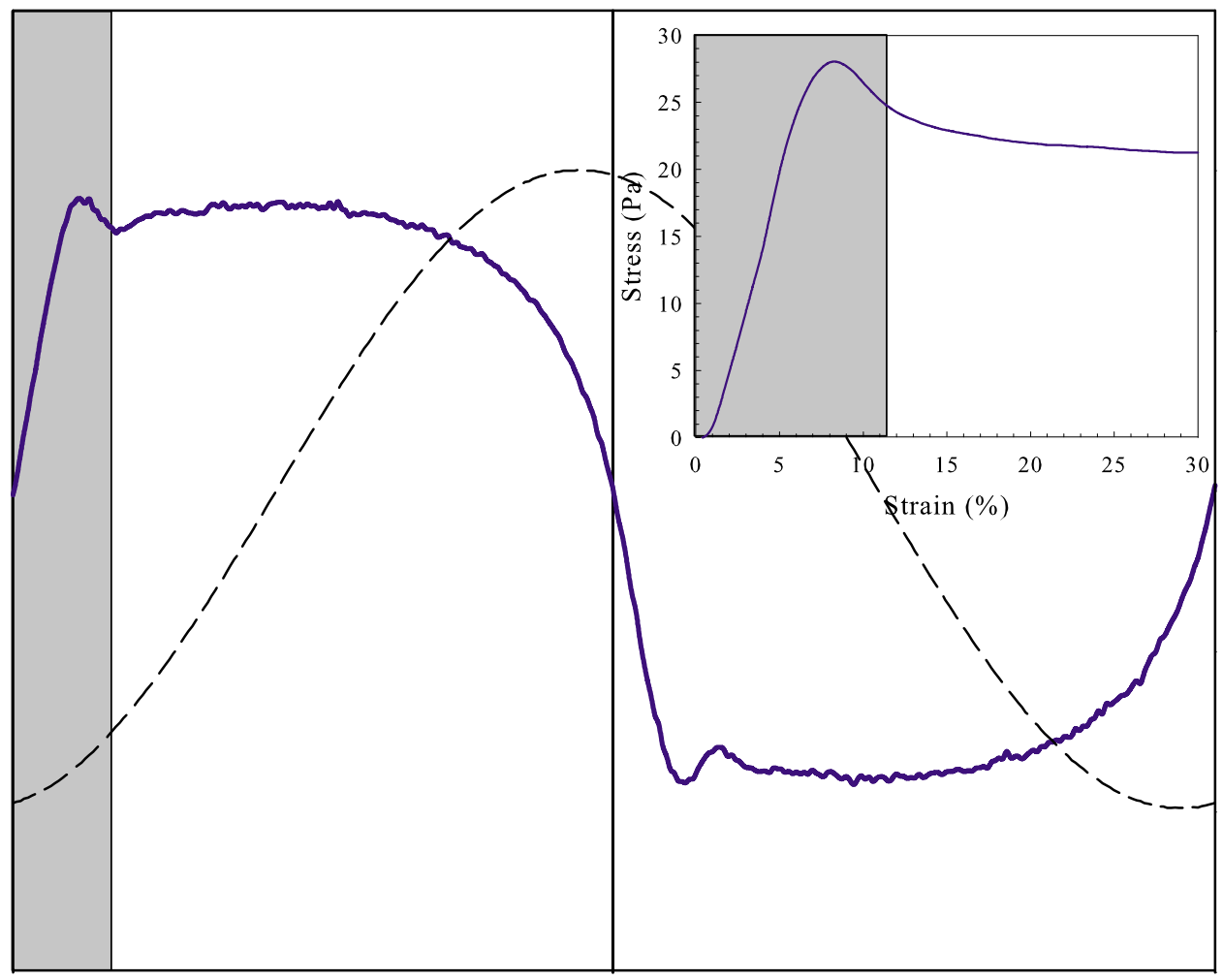

FIG. 10. Shape of the stress response (solid blue line) for $100 \%$ strain (dash line) and $\omega=10 \mathrm{rad} / \mathrm{s}$ over one period for a solution of PEP-PEO at $\mathrm{T}=10^{\circ} \mathrm{C}\left(c=2.64 c^{*}\right)$. The gray region represents the elastic dominant region and corresponds to the same accumulated strain $(\sim 12 \%)$ as that in the step rate test of the inset. The white regions represent the viscous dominant stress response over the positive stress half period. The inset depicts a step rate experiment for the same system at a rate of $0.5 \mathrm{~s}^{-1}$. The gray and the white regions have the same meaning as in the main plot.

contribution from Brownian motion that may relax shear induced stresses faster than stored. The decay of stress beyond the maximum strain corresponds to the cage reformation since when the strain reaches its maximum the strain rate is minimized.

The mechanical response of the present soft colloid glass under LAOS can be attributed to three distinct microscopic mechanisms within half a period of oscillation, which is then replicated at the subsequent half when strain is reversed: (i) initially the cage is distorted elastically for strains below the yield strain allowing a stress build up (elastic response); (ii) then at strains around the yield strain (about 10\%), several cages break leading to stress relaxation; (iii) when the yield strain is exceeded the glass is shear melted with continuous breaking and reformation of cages (viscous response). Finally, after the rate is minimized and the strain is reversed, cages have reformed and the reverse procedure starts. Note that the cage breaking picture during yielding of a colloidal glass has been evidenced by light scattering and microscopy measurements both under oscillatory and steady shear [Petekidis et al. (2002, 2003, 2004); Besseling et al. (2007)].

The interplay of shear with Brownian motion is represented at high volume fractions by the "dressed" Péclet number, $\mathrm{Pe}=\dot{\gamma} R^{2} / D_{s}(\phi)$, where $D_{s}(\phi)=D_{0} f(\phi)$ is the concentration dependent short-time diffusion coefficient and $D_{0}=k T / 6 \pi \eta R$ is the Stokes-EinsteinSutherland diffusivity of a particle with radius $\mathrm{R}$ in a medium with viscosity $\eta$. In 
oscillatory shear, a measure of the average shear rate is $\dot{\gamma}=\gamma_{0} \omega$. At low strain rates, i.e., at low frequencies for a given imposed strain above the yield strain, Pe may still be small enough that Brownian motion prevails over shear in a way that cage escape is enhanced by particle diffusion under shear. At higher frequencies, shear effects become more pronounced and at $\mathrm{Pe} \sim 1$ are equally important with Brownian motion with the former pulling the particles out of their cage, while the latter relaxes stresses and reforms cages. Similarly during start-up flow at $\mathrm{Pe} \sim 1$ or larger, stress overshoots are observed since shear can distort cages before Brownian motion relaxes them back. Moreover, for the present soft hairy micelles, arm interpenetration, absent in hard spheres, may cause arm stretching in a maximally distorted cage. Both these effects would increase the elasticity of the cages yielding an intracycle strain hardening. The latter could be clarified by a quantitative comparison with similar studies in hard sphere glasses.

In summary, using an appropriate decomposition of the stress, we have determined the relative and absolute strain hardening/softening and shear thickening/thinning contributions, suggesting that specific intracycle processes at a length-scale of the cage size dictate the nonlinear rheology of the present soft colloidal glass. Besides the simplistic classification of the response in a dynamic strain sweep in two regions, below and above the $G^{\prime}-G^{\prime \prime}$ crossover, here we clearly demonstrate that during such tests four different regimes exist with regard to the character of the dominant nonlinear response, as noted in Figs. 2(b), 7, and 8. In this context, the terms elastoviscous (or viscoelastic solid) when $G^{\prime}>G^{\prime \prime}$ (regions I and II) and viscoelastic (or viscoelastic liquid) when $G^{\prime \prime}>G^{\prime}$ (regions III and IV) may be used. In region I, the sample exhibits a linear elastoviscous behavior since $G^{\prime}>G^{\prime \prime}$ and nonlinearities are essentially absent. In region II, $G^{\prime}$ is still larger than $G^{\prime \prime}$ but nonlinearities set in revealing an intracycle shear thickening contribution. This region corresponds to a viscous modulated elastoviscous behavior. This shear thickening intracycle contribution appears prior to and around the macroscopic yield strain of the glass, where $G^{\prime \prime}$ and thus energy dissipation are maximized. It corresponds to the strain where the cage confinement starts to be important; i.e., particles start to feel their constricting cage under shear. Note that in this region the average mechanical behavior is still mainly elastic, whereas the nonlinear properties are dominated by a viscous stress response.

Then above the $G^{\prime}-G^{\prime \prime}$ crossover (about 7\% strain) and up to about $50 \%$ strain (region III), the nonlinear contribution starts to be strain hardening with the sample showing an elastically modulated viscoelastic behavior due to the cage breaking and stress relaxation. Thus, the nonlinearity is elastically modulated although the average macroscopic rheology is viscous-like. Finally, in region IV, a viscous modulated viscoelastic behavior is observed where the soft glass exhibits on average the mechanical response of a shear thinning fluid with intracycle nonlinearities of the same character. In regions III and IV, the system on average has been shear melted with $G^{\prime \prime}>G^{\prime}$ but the determination of the nonlinearities allows a more detailed investigation of intracylce yielding.

\section{CONCLUSIONS}

We investigated the nonlinear mechanical response of soft particle glasses under LAOS. Lissajous plots allow for a visual overall inspection of the nonlinear behavior at different strains and frequencies. FT analysis and decomposition in orthogonal Chebyshev polynomials of the stress response suggest the existence of distinct physical mechanisms within the oscillatory cycle responsible for such nonlinearities. The phenomenology of such intracycle response is classified according to the predominant Chebyshev 
polynomial coefficients into strain hardening/softening and shear-thickening/thinning when the elastic or viscous component, respectively, prevails. More specifically we observed that the intracycle character of nonlinearities exhibits a smooth transition from shear thickening to strain hardening to shear thinning with increasing strain. We should stress, however, that although the above phenomenological classification of nonlinear intracycle response during LAOS seems quite robust, the underlying physical mechanisms are only now starting to be explored. In this sense, the intracycle nonlinearity is expected to involve processes such as cage breaking and relaxation, viscous flow, and cage reformation due to Brownian motion. In conclusion, we note that in order to directly relate such phenomenology of the nonlinear rheology to detailed microscopic mechanisms and particle rearrangements, simultaneous rheometry and microscopy or scattering, on the one hand, and computer simulations, on the other hand, would be necessary.

\section{ACKNOWLEDGMENTS}

We thank Nikos Koumakis and Andreas Poulos for valuable discussions and technical assistance and Simon Rogers for fruitful discussions. We also acknowledge Randy Ewoldt for providing the MITLAOS software for the Chebyshev decomposition. This work has been supported by the EU funding through NoE Softcomp, ToK Cosines, and NMP SMALL Nanodirect. J.S. acknowledges DFG for support via SFB-TR6.

\section{References}

Atalık, K., and R. Keunings, "On the occurrence of even harmonics in the shear stress response of viscoelastic fluids in large amplitude oscillatory shear," J. Non-Newtonian Fluid Mech. 122, 107-116 (2004).

Besseling, R., E. R. Weeks, A. B. Schofield, and W. C. K. Poon, "Three-dimensional imaging of colloidal glasses under steady shear," Phys. Rev. Lett. 99, 028301 (2007).

Carrier, V., and G. Petekidis, "Nonlinear rheology of colloidal glasses of soft thermosensitive microgel particles," J. Rheol. 53(2), 245-273 (2009).

Cho, K. S., K. Hyun, K. H. Ahn, and S. J. Lee, "A geometrical interpretation of large amplitude oscillatory shear response," J. Rheol. 49, 747-758 (2005).

Christopoulou, C., G. Petekidis, B. Erwin, M. Cloitre, and D. Vlassopoulos, "Ageing and yield behavior in model soft colloidal glasses,” Philos. Trans. R. Soc. Ser. A 367, 5051-5071 (2009).

Cipelletti, L., H. Bissig, V. Trappe, P. Ballesta, and S. Mazoyer, "Time-resolved correlation: A new tool for studying temporally heterogeneous dynamics," J. Phys.: Condens. Matter 15, S257-S262 (2003).

Cloitre, M., R. Borrega, F. Monti, and L. Leibler, "Glassy dynamics and flow properties of soft colloidal pastes," Phys. Rev. Lett. 90, 068303 (2003).

Craciun, L., P. J. Carreau, M.-C. Heuzey, T. G. M. van de Ven, and M. Moan, "Rheological properties of concentrated latex suspensions of poly(styrene-butadiene)," Rheol. Acta 42, 410-420 (2003).

Deike, I., M. Ballauff, N. Willenbacher, and A. Weiss, "Rheology of thermosensitive latex particles including the high-frequency limit," J. Rheol. 45, 709-720 (2001).

Derec, C., G. Ducouret, A. Ajdari, and F. Lequeux, "Aging and nonlinear rheology in suspensions of polyethylene oxide-protected silica particles,” Phys. Rev. E 67, 061403 (2003).

Duri, A., H. Bissig, V. Trappe, and L. Cipeletti, "Time-resolved-correlation measurements of temporally heterogeneous dynamics," Phys. Rev. E 72, 051401 (2005).

El Masri, D., G. Brambilla, M. Pierno, G. Petekidis, A. B. Schofield, L. Berthier, and L. Cipelletti, "Dynamic light scattering measurements in the activated regime of dense colloidal hard spheres," J. Stat. Mech.: Theory Exp. 2009, P07015 (2009).

Ewoldt, R. H., A. E. Hosoi, and G. H. McKinley, "New measures for characterizing nonlinear viscoelasticity in large amplitude oscillatory shear,” J. Rheol. 52(6), 1427-1458 (2008). 
Fielding, S. M., P. Sollich, and M. E. Cates, “Aging and rheology in soft materials," J. Rheol. 44(2), 323-369 (2000).

Fuchs, M., and M. E. Cates, "Schematic models for dynamic yielding of sheared colloidal glasses," Faraday Discuss. 123, 267-286 (2003).

Fuchs, M., and M. E. Cates, "A mode coupling theory for Brownian particles in homogenous shear flow," J. Rheol. 53(4), 957-1000 (2009).

Gadala-Maria, F., and A. Acrivos, "Shear-induced structure in a concentrated suspension of solid spheres," J. Rheol. 24(6), 799-814 (1980).

Graham, M. D., "Wall slip and the nonlinear dynamics of large amplitude oscillatory shear flows," J. Rheol. 39(4), 697-712 (1995).

Hamley, I. W., The Physics of Block Copolymers (Oxford University Press, New York, 1998).

Helgeson, M. E., N. J. Wagner, and D. Vlassopoulos, "Viscoelasticity and shear melting of colloidal star polymer glasses,” J. Rheol. 51, 297-316 (2007).

Hyun, K., J. G. Nam, M. Wilhellm, K. H. Ahn, and S. J. Lee, "Large amplitude oscillatory shear behavior of PEO-PPO-PEO triblock copolymer solutions,” J. Rheol. 45, 239-249 (2006).

Kallus, S., N. Willenbacher, S. Kirsch, D. Distler, T. Neidhöfer, M. Wilhelm, and H. W. Spiess, "Characterisation of polyemer dispersions by Fourier transform rheology," Rheol. Acta 40, 552-559 (2001).

Kapnistos, M., D. Vlassopoulos, G. Fytas, K. Mortensen, G. Fleischer, and J. Roovers, "Reversible thermal gelation in soft spheres," Phys. Rev. Lett. 85(19), 4072-4075 (2000).

Koumakis, N., A. B. Schofield, and G. Petekidis, "Effects of shear induced crystallization on the rheology and ageing of hard sphere glasses," Soft Matter 4(10), 2008-2018 (2008).

Laurati, M., J. Stellbrink, R. Lund, L. Willner, E. Zaccarelli, and D. Richter, “Asymmetric poly(ethylene-altpropylene)-poly(ethylene oxide) micelles: A system with starlike morphology and interactions," Phys. Rev. E 76, 041503 (2007).

Laurati, M., J. Stellbrink, R. Lund, L. Willner, D. Richter, and E. Zaccarelli, "Starlike micelles with starlike interactions: A quantitative evaluation of structure factors and phase diagram," Phys. Rev. Lett. 94, 195504 (2005).

Le Grand, A., and G. Petekidis, "Effects of particle softness on the rheology and yielding of colloidal glasses," Rheol. Acta 47, 579-590 (2008).

Liddel, P. V., and D. V. Boger, "Yield measurements with the vane," J. Non-Newtonian Fluid Mech. 63(2-3), 235-261 (1996).

Likos, C. N., "Soft matter with soft particles," Soft Matter 2, 478-498 (2006).

Lonetti, B., J. Kohlbrecher, L. Wilner, J. K. Dhont, and M. P. Lettinga, "Dynamic response of block copolymer wormlike micellles to shear flow,” J. Phys.: Condens. Matter 20, 404207 (2008).

Loppinet, B., G. Fytas, D. Vlassopoulos, C. N. Likos, G. Meier, and G. J. Liu, "Dynamics of dense suspensions of star-like micelles," Macromol. Chem. Phys. 206, 163-172 (2005).

Loppinet, B., E. Stiakakis, D. Vlassopoulos, G. Fytas, and J. Roovers, "Reversible thermal gelation in star polymers: An alternative route to jamming of soft matter," Macromolecules 34, 8216-8223 (2001).

Lund, R., L. Willner, and D. Richter, "Equilibrium chain exchange kinetics of diblock copolymer micelles: Tuning and logarithmic relaxation," Macromolecules 39(13), 4566-4575 (2006).

Lund, R., L. Willner, J. Stellbrink, A. Radulescu, and D. Richter, "Role of interfacial tension for the structure of PEP-PEO polymeric micelles. A combined SANS and pendant drop tensiometry investigation," Macromolecules 37, 9984-9993 (2004).

Mason, T. G., J. Bibette, and D. A. Weitz, "Yielding and flow of monodisperse emulsions," J. Colloid Interface Sci. 179, 439-448 (1996).

Mason, T. G., M.-D. Lacasse, G. S. Grest, D. Levine, J. Bibette, and D. A. Weitz, "Osmotic pressure and viscoelastic shear moduli of concentrated emulsions," Phys. Rev. E 56, 3150-3166 (1997).

Miyazaki, K., H. M. Wyss, D. A. Weitz, and D. R. Reichman, "Nonlinear viscoelasticity of metastable complex fluids," Europhys. Lett. 75(6), 915-921 (2006).

Mortensen, K., E. Theunissen, R. Kleppinger, K. Almdal, and H. Reynaers, "Shear-induced morphologies of cubic ordered block copolymer micellar networks studies by in situ small-angle neutron scattering and rheology," Macromolecules 35, 7773-7781 (2002). 
Neidhöfer, T., M. Wilhelm, and B. Debbaut, "Fourier-transform rheology experiments and finite-element simulations on linear polystyrene solutions," J. Rheol. 47(6), 1351-1371 (2003).

Nicolai, T., and L. Benyahia, "Shear flow and large strain oscillation of dense polymeric micelle suspension," Macromolecules 38, 9794-9802 (2005).

Petekidis, G., A. Moussaïd, and P. N. Pussey, "Rearrangements in hard-sphere glasses under oscillatory shear strain,” Phys. Rev. E 66, 051402 (2002).

Petekidis, G., D. Vlassopoulos, and P. N. Pussey, "Yielding and flow of shear colloidal glasses," J. Phys.: Condens. Matter 16, S3955 (2004).

Petekidis, G., D. Vlassopoulos, and P. N. Pussey, "Yielding and flow of colloidal glasses," Faraday Discuss. 123, 287 (2003).

Pham, K. N., G. Petekidis, and D. Vlassopoulos, "Yielding behavior of repulsion and attraction dominated colloidal glasses,” J. Rheol. 52(2), 649-676 (2008).

Pham, K. N., G. Petekidis, D. Vlassopoulos, S. U. Egelhaaf, P. N. Pusey, and W. C. K. Poon, "Yielding of colloidal glasses," Europhys. Lett. 75, 624-630 (2006).

Pham, K. N., A. M. Puertas, J. Bergenholtz, S. U. Egelhaaf, A. Moussaïd, P. N. Pusey, A. B. Schofield, M. E. Cates, M. Fuchs, and W. C. K. Poon, "Multiple glassy states in a simple model system," Science 296, 104-106 (2002).

Philippoff, W., "Vibrational measurements with large amplitudes," J. Rheol. 10, 317-334 (1966).

Poppe, A., L. Willner, J. Allgaier, J. Stellbrink, and D. Richter, "Structural investigation of micelles formed by an amphiphilic PEP-PEO block copolymer in water," Macromolecules 30(24), 7462-7471 (1997).

Poulos, A., N. Koumakis, et al., unpublished.

Purnomo, E. H., D. van den Ende, S. A. Vanapalli, and F. Mugele, "Glass transition and aging in dense suspensions of thermosensitive microgel particles," Phys. Rev. Lett. 101, 238301 (2008).

Pusey, P. N., in Liquids, Freezing and the Glass Transition, Les Houches, edited by J. P. Hansen, D. Levesque, J. Zinn-Justin, and L. I. Session (Elsevier, Amsterdam, 1991).

Pusey, P. N., and W. Van Megen, "Phase behaviour of concentrated suspensions of nearly hard colloidal spheres," Nature (London) 320, 340 (1986).

Pusey, P. N., and W. Van Megen, "Observation of glass transition in suspensions of spherical colloidal particles," Phys. Rev. Lett. 59, 2083 (1987).

Renou, F., L. Benyahia, and T. Nicolai, "Influence of adding linear polymer on liquid-solid transition of dense polymeric micelle solutions," Macromolecules 40(13), 4626-4634 (2007).

Renou, F., L. Benyahia, and T. Nicolai, "Structure and rheology of mixed micelles formed by hydrophobically end-capped poly(ethylene oxide)," Macromolecules 41, 6523-6530 (2008).

Renou, F., T. Nicolai, E. Nicol, and L. Benyahia, "Structure and viscoelasticity of mixed micelles formed by poly(ethylene oxide) end-capped with alkyl groups of different length," Langmuir 25(1), 515-521 (2009).

Rogers, S. A., and D. Vlassopoulos, "Frieze group analysis of asymmetric response to large amplitude oscillatory shear," J. Rheol. 54(4), 859-880 (2010).

Roovers, J., P. M. Toporowski, and J. Douglas, "Thermodynamic properties of dilute and semi-dilute solutions of regular star polymers," Macromolecules 28, 7064-7070 (1995).

Rouyer, F., S. Cohen-Addad, R. Höhler, P. Sollich, and S. M. Fielding, "The large amplitude oscillatory strain response of aqueous foam: Strain localization and full stress Fourier spectrum,” Eur. Phys. J. E 27, 309-321 (2008).

Seghrouchni, R., G. Petekidis, D. Vlassopoulos, G. Fytas, A. N. Semenov, J. Roovers, and G. Fleischer, "Controlling the dynamics of soft spheres: From polymeric to colloidal behavior," Europhys. Lett. 42(3), 271-276 (1998).

Senff, H., W. Richtering, Ch. Norhausen, A. Weiss, and M. Ballauff, "Rheology of temperature sensitive core-shell latex," Langmuir 15, 102-106 (1999).

Seth, J. R., M. Cloitre, and R. T. Bonnecaze, "Elastic properties of soft particle pastes," J. Rheol., 50(3), 353-376 (2006).

Siebenbürger, M., M. Fuchs, and H. Winter, "Viscoelasticity and shear flow of concentrated, noncrystallizing colloidal suspensions: Comparison with mode-coupling theory," J. Rheol. 53(3), 707-726 (2009).

Sollich, P., "Rheological constitutive equation for a model of soft glassy materials," Phys. Rev. E 58(1), 
738-759 (1998).

Sommer, C., and J. S. Pederson, "Temperature dependence of the structure and interaction of starlike PEGbased block copolymer micelles," Macromolecules 37, 1682-1685 (2004).

Stellbrink, J., B. Lonneti, G. Rother, L. Willner, and D. Richter, "Shear induced structures of soft colloids: Rheo-SANS experiments on kinetically frozen PEP-PEO diblock copolymer micelles," J. Phys.: Condens. Matter 20, 404206 (2008).

Stiakakis, E., D. Vlassopoulos, C. N. Likos, J. Roovers, and G. Meier, "Polymer-mediated melting in ultrasoft gels," Phys. Rev. Lett. 89(20), 208302 (2002).

Stokes, J. R., and J. H. Telfor, "Measuring the yield behaviour of structured fluids," J. Non-Newtonian Fluid Mech. 124(1-3), 137-146 (2004).

Tee, T. T., and J. M. Dealy, "Nonlinear viscoelasticity of polymer melts," J. Rheol. 19, 595-615 (1975).

van Ruymbeke, E., A. Pamvouxoglou, D. Vlassopoulos, G. Petekidis, G. Mountrichas, and S. Pispas, "Rheological behaviour of stable, responsive diblock copolymer micelles," Soft Matter 6, 881-891 (2010).

Watanabe, H., Y. Matsumiya, T. Kanaya, and Y. Takahashi, "Rheology and structure of a butadiene-styrene dibloc copolymer in dibutyl phtalate: Role of concentration fluctuation in disruption and reformation of micellar lattice," Macromolecules 34, 6742-6755 (2001).

Watanabe, H., T. Sato, and K. Osaki, "Rheological and dielectric behavior of a stryrene-isoprene-styrene triblock copolymer in selective solvents. 2. Contribution of loop-type middle blocks to elasticity and plasticity," Macromolecules 30, 5877-5892 (1997).

Wilhelm, M., D. Maring, and H.-W. Spiess, "Fourier-transform rheology," Rheol. Acta 37, 399-405 (1998).

Wilhelm, M., P. Reinheimer, M. Ortseifer, T. Neidhofer, and H.-W. Spiess, "The crosseover between linear and non-linear mechanical behaviour in polymer solutions as detected by Fourier-transform rheology," Rheol. Acta 39, 241-246 (2000).

Willner, A., A. Poppe, J. Allgaier, M. Monkenbusch, and D. Richter, "Time-resolved SANS for the determination of unimer exchange kinetics in block copolymer micelles," Europhys. Lett. 55, 667-673 (2001).

Winter, H. H., and M. Mours, "Rheology of polymers near liquid-solid transitions," Adv. Polym. Sci. 134, 166-234 (1997).

Yamazaki, R., K. Inomata, and T. Nose, "Rheological properties of micellar aqueous solution of hydrophobically modified poly(ethylene glycol) (C12E25)," Macromol. Chem. Phys. 203, 2322-2328 (2002).

Zaccarelli, E., "Colloidal gels: Equilibrium and non-equilibrium routes,” J. Phys.: Condens. Matter 19, 323101 (2007). 